\title{
Numerical modeling of wave-mud interaction using projection method
}

\author{
Kourosh Hejazi • Mohsen Soltanpour • Saeideh Sami
}

Received: 30 April 2012 / Accepted: 18 June 2013 / Published online: 2 August 2013

(C) The Author(s) 2013. This article is published with open access at Springerlink.com

\begin{abstract}
A numerical model has been developed to simulate wave-mud interaction. The fully non-linear NavierStokes equations with complete set of kinematic and dynamic boundary conditions at free surface and interface with the two-equation $k-\varepsilon$ turbulence model with buoyancy terms are solved. Finite volume method based on an ALE description has been utilized for the simulation of wave motion in a combined system of water and viscous mud layer. The model is an extension to Width Integrated Stratified Environments 2DV numerical model, originally developed by Hejazi (2005). For validation of the hydrodynamics of the model, small-amplitude progressive wave train in deep water and solitary wave propagation in a constant water depth have been simulated, and the results have been compared with analytical solutions, which show very good agreements. A non-linear short wave propagation in a constant water depth has also been simulated, and the predictions have been compared against measured values reported in the literature, which confirms the model ability in prediction of non-linear short waves. Application of the new model in a combined
\end{abstract}

Responsible Editor: Andrew James Manning

This article is part of the Topical Collection on the 11th International Conference on Cohesive Sediment Transport

K. Hejazi $\cdot$ M. Soltanpour $\cdot$ S. Sami $(\bowtie)$

Department of Civil Engeneering, K. N. Toosi University

of Technology, No.1346, Vali-Asr Ave.

P.C. 1996715433, Tehran, Iran

e-mail: sami@dena.kntu.ac.ir

K. Hejazi

e-mail: hejazik@kntu.ac.ir

URL: http://ihr.ir

M. Soltanpour

e-mail: soltanpour@kntu.ac.ir

URL: http://sahand.kntu.ac.ir/ soltanpour/ system of viscous fluid mud shows good agreements in determining damping coefficient and water-mud interface elevation for various wave heights and frequencies compared to the experimental data. Simulated surface wave number values obtained for various mud layer thicknesses show very good agreements compared with analytical solution results.

Keywords Wave-mud interaction · Fluid mud · Projection method · ALE description · WISE 2DV numerical model . $\mathrm{FVM} \cdot$ Buoyant $k$ - $\varepsilon$ turbulence model

\section{Introduction}

Some coastal and most estuarine sea beds are loaded with cohesive sediments. In the presence of cohesive sediments, wave damping is enhanced; surface waves can be attenuated appreciably in a finite number of wave periods or wave lengths. Meanwhile, the waves can induce a Lagrangian drift on the bottom, driving a slow but steady mass transport of the mud. Cohesive sediment can be considered to exist in four states: a mobile suspended sediment, a high concentration near the bed layer which sometimes is referred to as fluid mud, a newly deposited or partially consolidated bed, and a settled or consolidated bed. The upper layer of suspension is separated by a sharp density gradient or lutocline from the fluid mud (Whitehouse et al. 2000). Suspended sediments have the largest mobility and may travel long distances before depositing. In contrast, the highly concentrated fluid mud, which tends to exhibit profound non-Newtonian behavior, is much limited in motion. Nevertheless, the fluid mud is a complicated layer which plays a crucial role compared to other layers in wave-mud interaction. The fluid mud 
may be developed either by fast deposition of suspended sediment or by fluidization or failure of soft, freshly deposited mud layers at sea bed (Mehta et al. 1995 and Li and Mehta 1997). Extensive studies have been carried out to understand the mechanism of mud and water in a wavedominated marine environment, whereas most of them focus on fluid mud layer as sediment layer.

Since the late 1950s and after Gade (1958), a relatively large number of analytical and experimental works have been carried out. Most of the analytical works hold the assumption of a Newtonian fluid to take advantage of linearizing the stress and rate of strain relations. The equations of motion are the laminar Navier-Stokes equations, which have been linearized by neglecting the advective accelerations (e.g., Dalrymple and Liu 1978 and Maa and Mehta 1990). In the case of complete Navier-Stokes equations, a perturbation analysis may be carried out to the first and second order to reveal the mean Lagrangian drift in two layers (e.g., Ng 2000 and Ng 2004).

In spite of an extensive number of analytical studies, very few numerical model developments for wave-mud interaction have been reported in the literature. For instance, Zhang and $\mathrm{Ng}$ (2006) presented a numerical model for a two-layer viscous fluid system to simulate a progressive wave in the upper layer and the oscillatory motion of lower mud layer induced by the water wave to evaluate the ratio of interfacial to surface wave amplitude. They used the dimensionless conservative form of time-dependent Navier-Stokes equations in a curvilinear coordinate system fitted to the moving interfaces. Governing equations were solved, and the boundary conditions were implemented by a time-splitting fractional step method in a two-step predictor-corrector scheme in a finite difference fashion. An intermediate velocity field was computed at the prediction step, and the location of the free surface and the interface was evaluated afterwards. Within a time step, the solution procedure was first applied to the upper layer, and then by the application of the boundary conditions across the fluid interface, the solution procedure was applied to the lower layer. Niu and Yu (2010) developed their model based on the well-known simplified marker and cell method using a finite difference scheme, in which the motion of the movable mud and water were solved simultaneously. Water was treated as a viscous fluid, while a visco-elastic-plastic model was considered for the mud layer. The free surface and the interface were both traced by the volume of fluid method. Their model was applied to simulate wave propagation over a muddy slope.

Propagation of surface water waves over a mud layer generates an interfacial wave between the water and mud layer, resulting in a high wave energy dissipation in comparison with non-cohesive sediments. This paper presents the development of a numerical model to simulate the wave motion and wave-mud interaction in a two-phase viscous fluid system. Surface wave height attenuation and interfacial wave amplitude are investigated. To address these, the damping coefficient of surface waves is computed for various initial wave heights and frequencies, and surface wave number has been calculated for alternative mud layer thicknesses. The upper layer of the system consists of plain water subject to a surface wave disturbance, while the lower mud layer is treated as a viscous fluid characterized by a viscosity and density greater than the water layer. The new model is an extension to WISE (Width Integrated Stratified Environments) 2DV (two dimensional vertical) hydrodynamic model. WISE is a finite volume method non-hydrostatic Reynolds-averaged Navier-Stokes (RANS) free-surface numerical model using a structured non-orthogonal curvilinear staggered mesh and is capable of simulating non-homogeneous, gravity-stratified flow fields. The two-equation $k-\varepsilon$ turbulence model with buoyancy terms has been included in the numerical model. Projection (fractional step) method has been used for solving the equations. In WISE, the free surface equation is obtained by integrating the continuity equation over depth with the application of kinematic boundary conditions at bed and free surface (Hejazi 2005). In the refined model, for improving the free surface prediction, which plays a crucial role in wave-mud interaction simulation, the dynamic free surface boundary condition has been modified according to the method of Ahmadi et al. (2007). To simulate wave-mud interaction, the original hydrodynamic model has also been modified to include mud and consideration of interface boundary conditions for the simulation of two-layer fluid mud system and prediction of water-mud interface elevation. To provide flexibility and higher resolution for prediction of mud surface, gridding has been adjusted accordingly, and the model has been modified for accommodating non-uniform gridding in a vertical direction in two layers.
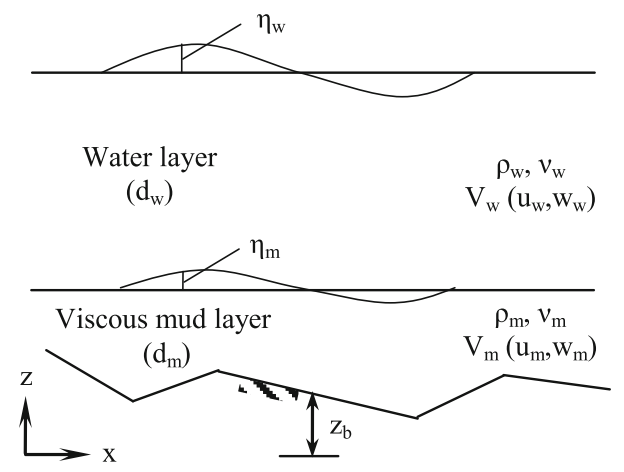

Fig. 1 Schematic diagram of the two-layer viscous fluid system in the 2DV presentation 


\section{Governing equations, and spatial and temporal boundary conditions}

The governing equations of the 2DV problem in arbitrary Lagrangian-Eulerian (ALE) description together with the buoyant $k-\varepsilon$ turbulence model have been presented herein. Spatial and temporal boundary conditions of the solution domain have been addressed and discussed.

\subsection{Governing equations}

Figure 1 shows the physical domain bounded by the moving free surface, $\eta(x, t)$, and the bottom boundary, $z=z_{b}(x)$. The upper layer is plain water subject to a wave disturbance and the lower layer is fluid mud.

For incompressible flows in the two-dimensional vertical plane, and in the Cartesian coordinate system $(x, z, t)$, the conservative form of RANS equations in ALE description may be expressed in a compact vectorial form as (Tannehill et al. 1997):

$\frac{\partial V}{\partial t}+\frac{\partial F_{x}}{\partial x}+\frac{\partial F_{z}}{\partial z}=Q$

where $V, F_{x}, F_{z}$, and $Q$ are vectors given by:

$$
\begin{aligned}
& V=\left[\begin{array}{l}
0 \\
u \\
w
\end{array}\right] \\
& F_{x}=\left[\begin{array}{c}
u \\
u^{2}-\nu_{t} \partial u / \partial x+P^{*} \\
u w-\nu_{t} \partial w / \partial x
\end{array}\right] ; F_{z}=\left[\begin{array}{c}
w u-w_{g} u-\nu_{t} \partial u / \partial z \\
w^{2}-w_{g} w-\nu_{t} \partial w / \partial z+P^{*}
\end{array}\right] ; Q=\left[\begin{array}{c}
0 \\
0 \\
-g\left(\rho-\rho_{\mathrm{r}}\right) / \rho_{\mathrm{r}}
\end{array}\right]
\end{aligned}
$$

where $t$ is time; $x$ and $z$ are coordinates in horizontal and vertical directions, respectively; $u$ and $w$ are components of velocity in the $x$ - and $z$-directions, respectively; $P^{*}$ is the pressure in the absence of hydrostatic pressure divided by the reference density of water; $\rho$ is the density of water; $\rho_{\mathrm{r}}$ is the reference density of water; $g$ is the gravitational acceleration; $v_{t}$ is the eddy viscosity coefficient; and $w_{g}$ is the mesh velocity obtained from the vertical displacement of mesh in each time step. In the ALE method, the newly updated free surface is determined purely by the Lagrangian method, by the velocity of the fluid particles at the free surface, while the nodes in the interior of the domain are displaced in an arbitrary prescribed manner to be redistributed to avoid mesh crossing.

The first row of Eq. (2) corresponds to the continuity equation, and the second and third rows represent the components of momentum equation in $x$ - and $z$-directions, respectively.

To optimize accuracy and economy, the two-equation $k-\varepsilon$ turbulence model with buoyancy terms has been deployed and included in the numerical model. The conservative form of the $k$ - $\varepsilon$ equations in ALE description is as follows:

$$
\nu_{t}=c_{\mu} \frac{k^{2}}{\varepsilon}
$$

$$
\frac{\partial k}{\partial t}+\frac{\partial u k}{\partial x}+\frac{\partial w k}{\partial z}-w_{g} \frac{\partial k}{\partial z}=\frac{\nu_{t}}{\sigma_{k}}\left(\frac{\partial^{2} k}{\partial x^{2}}+\frac{\partial^{2} k}{\partial z^{2}}\right)+P+G^{-\varepsilon}
$$

$$
\begin{aligned}
\frac{\partial \varepsilon}{\partial t}+\frac{\partial u \varepsilon}{\partial x} & +\frac{\partial w \varepsilon}{\partial z}-w_{g} \frac{\partial \varepsilon}{\partial z}=\frac{\nu_{t}}{\sigma_{\varepsilon}}\left(\frac{\partial^{2} \varepsilon}{\partial x^{2}}+\frac{\partial^{2} \varepsilon}{\partial z^{2}}\right) \\
& +c_{1 \varepsilon} \frac{\varepsilon}{k}\left(P+c_{3 \varepsilon} G\right)-c_{2 \varepsilon} \frac{\varepsilon^{2}}{k}
\end{aligned}
$$

where $k$ is kinematic energy, $\varepsilon$ is dissipation rate of energy, and the empirical constants $c_{\mu}, \sigma_{k}, c_{\varepsilon}, c_{1 \varepsilon}, c_{2 \varepsilon}$ and $c_{3 \varepsilon}$ are taken to be the same as those proposed by Rodi (1987). $P$ and $G$ are shear and buoyancy productions, respectively, and are given by:

$P=\nu_{t}\left[2\left(\frac{\partial u}{\partial x}\right)^{2}+2\left(\frac{\partial w}{\partial z}\right)^{2}+\left(\frac{\partial w}{\partial x}+\frac{\partial u}{\partial z}\right)^{2}\right]$

$G=\beta g \frac{\nu_{t}}{\sigma_{t}} \frac{\partial C}{\partial z}$

where $\sigma_{t}$ is the Schmidt number; $\beta$ is the compressibility coefficient of fluid, and $C$ is the species concentration, which may be obtained from the density.

\subsection{Spatial boundary conditions}

Spatial boundary conditions have been divided into five locations: the rigid bottom of the mud layer (bed), the interface boundary at plain water and mud flow, the free surface of water, the inlet, and the outlet boundaries. 


\subsubsection{Bed boundary condition}

The kinematic boundary condition at the impermeable bottom gives (Dean and Dalrymple 1991):

$u \frac{\partial z_{\mathrm{b}}}{\partial x}+w=0$

where $z_{\mathrm{b}}(x)$ is the bed elevation above datum.

\subsubsection{Free surface boundary conditions}

At the free surface, kinematic and two dynamic boundary conditions are applied.

Kinematic free surface boundary condition Within the Cartesian coordinate system, kinematic free surface boundary condition is formulated by the following one-dimensional hyperbolic wave equation (Dean and Dalrymple 1991):

$\frac{\partial \eta}{\partial t}+u \frac{\partial \eta}{\partial x}=w$

where $\eta(x, t)$ is the free surface elevation measured from the undisturbed mean water level. To keep the consistency, the free surface equation can be obtained by integrating continuity equation over depth and by the application of the kinematic conditions at bed (Eq. 8) and free surface (Eq. 9) as follows:

$\frac{\partial \eta}{\partial t}+\frac{\partial}{\partial x} \int_{z \mathrm{~b}}^{\eta} u_{f} d z=0, \quad f=m$ or $w$

in which $m$ stands for mud and $w$ stands for water.

Dynamic free surface boundary conditions The free surface dynamic conditions represent the equilibrium of the normal and the tangential components of the stresses on free surface. Neglecting the surface tension on free surface, the dynamic conditions are as follows:

$$
\sigma=0
$$$$
\tau=0
$$

where $\sigma$ and $\tau$ represent normal and tangential stress on free surface, respectively. Using the stress tensor $\left[\sigma_{i j}=-p \delta_{i j}+\mu\left(\frac{\partial u_{i}}{\partial x_{j}}+\frac{\partial u_{j}}{\partial x_{i}}\right)\right]$, Eq. (11) is written in the form of Eq. (12), which represents the normal and tangential dynamic boundary conditions, respectively (Hirt and Shannon 1968):

$$
\begin{aligned}
& p=\frac{2 \mu}{1+\left(\frac{\partial \eta}{\partial x}\right)^{2}}\left[\left(\frac{\partial \eta}{\partial x}\right)^{2} \frac{\partial u}{\partial x}-\left(\frac{\partial \eta}{\partial x}\right)\left(\frac{\partial u}{\partial z}+\frac{\partial w}{\partial x}\right)+\frac{\partial w}{\partial z}\right] \\
& 2\left(\frac{\partial \eta}{\partial x} \times \frac{\partial u}{\partial x}\right)+\left(\left(\frac{\partial \eta}{\partial x}\right)^{2}-1\right)\left(\frac{\partial u}{\partial z}+\frac{\partial w}{\partial x}\right)-2\left(\frac{\partial \eta}{\partial x} \times \frac{\partial w}{\partial z}\right)=0
\end{aligned}
$$

where $P$ represents the pressure at free surface and $\mu$ is the viscosity of water.

\subsubsection{Interface boundary conditions}

The interface boundary conditions provide no exchange (erosion or deposition) across the water-mud interface and may be represented in the same manner as the free surface boundary conditions. The kinematic interface boundary condition is as Eq. (13):

$\frac{\partial \eta_{\mathrm{m}}}{\partial t}+u_{f} \frac{\partial \eta_{\mathrm{m}}}{\partial x}=w_{f}, \quad f=m$ or $w$

where $\eta_{\mathrm{m}}(x, t)$ is the mud surface elevation measured from undisturbed mean mud level. The normal and tangential dynamic boundary conditions for the interface are written as follows (Zhang and Ng 2006):

$$
\begin{aligned}
& \sigma_{\mathrm{w}}=\sigma_{\mathrm{m}} \\
& \tau_{\mathrm{w}}=\tau_{\mathrm{m}}
\end{aligned}
$$

The balance of fluxes should also be applied at the interface:

$$
\begin{aligned}
& u_{\mathrm{w}}=u_{\mathrm{m}} \\
& w_{\mathrm{w}}=w_{\mathrm{m}}
\end{aligned}
$$

\subsubsection{Inlet and outlet boundary conditions}

At inlet and outlet boundaries, the velocity or pressure or water elevation may be regarded as known values depending on circumstances. For instance in the case of wave propagation on the free surface where a flap-type wavemaker is applied at the inlet, by assuming a sinusoidal motion for generated wave, the stroke $\left(S_{0}\right)$ at the water surface may be calculated by Eq. (16), given by Dean and Dalrymple (1991), to obtain the inlet velocity at the water surface (Eq. 17).

$\frac{H}{S_{0}}=\frac{4 \sinh \left(k_{\mathrm{L}} d_{\mathrm{w}}\right)}{\sinh \left(2 k_{\mathrm{L}} d_{\mathrm{w}}\right)+2 k_{\mathrm{L}} d_{\mathrm{w}}}\left[\sinh \left(k_{\mathrm{L}} d_{\mathrm{w}}\right)+\frac{1-\cosh \left(k_{\mathrm{L}} d_{\mathrm{w}}\right)}{k_{\mathrm{L}} d_{\mathrm{w}}}\right]$

$u=\frac{\omega S_{0}}{2} \sin (\omega t)$

where $H$ is the wave height, $k_{L}$ is the wave number, $d_{w}$ is the depth of water layer, and $\omega$ is the wave frequency. To provide a free exit for water at the outlet and maintain upstream control hydraulic condition, a zero dynamic pressure condition is set at the far end of the domain.

\subsection{5 $k$ and $\varepsilon$ boundary conditions}

On the free surface, Neuman boundary for $k$ and Dirichlet boundary for $\varepsilon$ are used and set to zero. Neuman boundary for $k$ and $\varepsilon$ is set to zero at the outlet. At the inlet boundary, it is assumed that the flow is smooth, and $k$ and $\varepsilon$ are set to small values different from zero. 


\subsection{Temporal boundary conditions}

Initial values are required for the water elevation and velocity components within the computational domain. The initial velocity and pressure values are set equal to zero. $k$ and $\varepsilon$ are set to suitable values to give an appropriate kinematic value for viscosity.

\section{Numerical method}

The model developed herein is an extension to WISE 2DV numerical model (Hejazi 2005). WISE uses a structured non-orthogonal curvilinear staggered mesh based on ALE description. The discretization of the flow and transport equations has been based on finite volume method, providing flexibility for defining control volumes in a staggered grid system, especially near the bed and water surface where rapid changes of the bathymetry and free surface may have a significant effect on the prediction of flow field. The finite volume method also provides, if correctly implemented, the assurance of global conservation. For modeling the turbulence phenomenon and to optimize accuracy and economy, the two-equation $k-\varepsilon$ turbulence model with buoyancy terms has been deployed in the numerical model. The model is also capable of simulating nonhomogeneous (i.e., variable density), gravity-stratified flow fields.

The projection of the geometry and grid configuration, the control volumes of the scalar and vector quantities, and their locations on the $x o z$ reference plane are illustrated in Fig. 2. The scalar variables including pressure, $k, \varepsilon$, viscosity, and density are calculated at the nodal points $(\bullet)$. The velocity components are calculated at the central point of each face of a scalar control volume drawn around the scalar quantities (e.g., pressure points) and are located midway between scalar quantities (e.g., pressure points). The velocity components are indicated by horizontal and vertical arrows for $u$-, and $w$ velocity components, respectively. The control volumes for the scalar quantities and $w$-velocity component consist of six sides, and the control volume for the $u$-velocity component consists of four sides. Surface and bottom elevations are defined at the center of the corresponding surface and bottom cells, respectively.

\subsection{Numerical approximation}

A finite volume approximation is used to discretize the governing equations and boundary conditions. The discretization of the vectorial Eq. (1) includes either the derivative of a quantity or the derivative of a flux. According to divergence theorem, the average $x$-derivative, for instance, of a quantity $\Phi$ for the representative control volumes of Fig. 2 is obtained from Eq. (18) (Hejazi 2005):

$\overline{\left(\frac{\partial \Phi}{\partial x}\right)_{\Omega}} \approx \frac{1}{\Omega_{\Re}} \sum_{\text {sides }} \Phi . \Delta z$

where $\Omega_{\Re}$ is the volume value of the finite volume $\mathfrak{R}$. Applying the above relationship to the control volumes of velocity components presented in Fig. 2, the $x$ derivative of the horizontal velocity and the $\mathrm{z}$ derivative of the vertical velocity for the grid $(I, K)$ may be written as:

$$
\begin{aligned}
& \left(\frac{\partial u}{\partial x}\right)_{I, K} \approx \frac{1}{A_{I, K}}\left[u_{I-1 / 2, K} \Delta z_{I-1 / 2}+\bar{u}_{I, K-1 / 2} \Delta z_{I}+u_{I+1 / 2, K} \Delta z_{I+1 / 2}+\bar{u}_{I, K+1 / 2} \Delta z_{I}\right] \\
& \left(\frac{\partial w}{\partial z}\right)_{I, K} \approx \frac{1}{A_{I, K}}\left[w_{I, K-1 / 2}+w_{I, K+1 / 2}\right] \Delta x
\end{aligned}
$$

where $\bar{u}$ is the average horizontal velocity obtained by using four neighboring points.

\subsection{Solution procedure}

The model has utilized the projection method or the method of fractional step proposed by Chorin (1968) and Temam (1969). The solution may generally be accomplished in two steps. The pressure gradient terms are omitted from the momentum equations in the first step, and the unsteady equations are advanced in time to obtain a provisional velocity field. In the second step, the provisional velocity is corrected by accounting for the pressure gradient and the continuity equation.

\subsubsection{First step}

The first fractional step, which includes the solution of advective and diffusive terms, consists of finding - providing that $V^{n}$ is known-an intermediate or provisional velocity $\left(V^{*}\right)$. The step is further split into two sub-fractional steps, enabling separate computations of the advective and diffusive terms. 


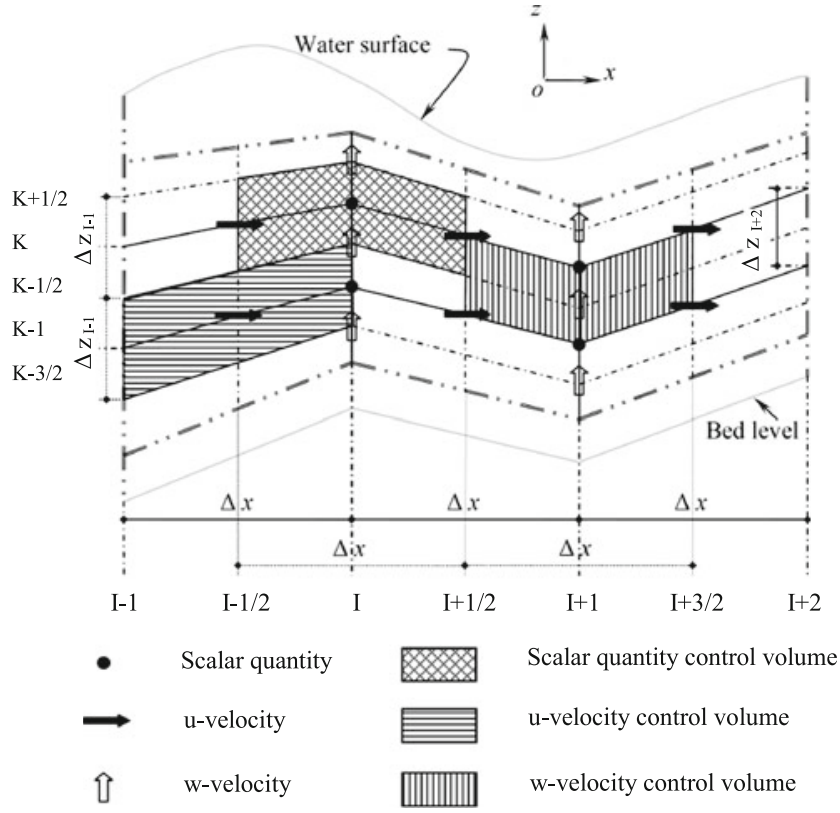

Fig. 2 Presentation of staggered grids, positions of the scalar and vector quantities, and the corresponding control volumes

This approach allows the use of the most suitable approximations for each term. Advection and diffusion are computed in a locally one-dimensional fashion. Therefore, the momentum equation in the absence of pressure gradient term is split into two equations which are computed sequentially as follows:

$\frac{V^{n \stackrel{A}{\rightarrow} n+1}-V^{n}}{\Delta t}+\operatorname{div}(V \otimes V)^{n}-w_{g}^{n}\left(\frac{\partial V}{\partial z}\right)^{n}=0$

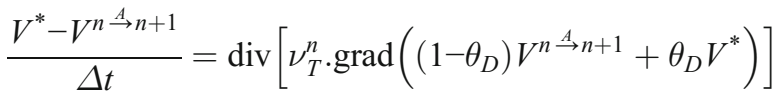

$A$ stands for advection, and its appearance denotes that the value corresponds to the time level when the advection process is completed, in the time interval $n \rightarrow n+1$. The advective contribution of the transport term in Eq. (20) is further split into three sub-subfractional steps. For large
Reynolds numbers, the flow is effectively advectiondominated (Weinan and Jian-Guo 1995); hence, to achieve more realistic predictions of the flow characteristics, the derivative approximation is obtained by assuming a fourthdegree polynomial as the shape function of the quantity to be advected, providing a fifth-order accurate scheme (Hejazi 2005).

Diffusion is computed by the Crank-Nicolson scheme with a weighing factor $\left(\theta_{D}\right)$ set to 0.5 (Eq. 21). The diffusion contribution also has been split into two sub-subfractional steps.

\subsubsection{Second step}

In the second step, by taking the divergence of Eq. (22), and subject to the continuity constraint (Eq. 23), the Poisson equation is obtained (Eq. 24):

$\frac{V^{n+1}-V^{*}}{\Delta t}+\nabla P^{* n+1}=0$

$\operatorname{div} V^{n+1}=0$

$\nabla^{2} P^{* n+1}=\frac{\operatorname{div} V^{*}}{\Delta t}$

This step makes use of the Hodge decomposition theorem, which states that any vector function can be decomposed into a divergence-free part, plus the gradient of a scalar potential (Brown 2001). The second step proceeds by solving the Poisson equation. In the second step, the pressure equation is obtained for each control volume of pressure in the domain except the boundary layers by the use of Eq. (24) for both water and mud layers.

\subsubsection{Poisson equation solver}

The pressure equation at free surface is obtained by using free surface equation (Eq. 10). The equation discretizes as follows:

$\frac{\eta_{I}^{n+1}-\eta_{I}^{n}}{\Delta t}+\frac{1}{\Delta x} \sum_{K=1}^{K_{\max }}\left[\theta \Delta z_{I+1 / 2} u_{I+1 / 2, K}^{n+1}-\theta \Delta z_{I-1 / 2} u_{I-1 / 2, K}^{n+1}+(1-\theta) \Delta z_{I+1 / 2} u_{I+1 / 2, K}^{n}-(1-\theta) \Delta z_{I-1 / 2} u_{I-1 / 2, K}^{n}\right]=0$

Applying fully non-hydrostatic pressure at the top layer, the calculated wave amplitude and phase are significantly improved and are well compared with the analytical solutions (Yuan and $\mathrm{Wu}$ 2004). For accurate prediction of free surface and to obtain the pressure equation, the top layer pressure gradient and vertical accelerations are treated implicitly, similar to the solution of the pressure equation in domain, by implementing free surface dynamic and kinematic boundary conditions (Ahmadi et al. 2007). Vertical momentum equation for column $I$ from the center of the top layer to the free surface 
is then approximated by Eq. (26):

$\frac{w_{I, T}^{n+1}-w_{I, T}^{*}}{\Delta t}+\varphi\left(\frac{P_{I, S}^{* n+1}-P_{I, K_{\max }^{*}}^{* n+1}}{\Delta z_{I} / 2}\right)+(1-\varphi)\left(\frac{P_{I, S}^{* n}-P_{I, K_{\max }}^{* n}}{\Delta z_{I} / 2}\right)=0$

where $P_{I, S}{ }^{* n+1}=g \eta_{I}{ }^{n+1}$ and $P_{I, S}{ }^{* n}=g \eta_{I}^{n}$ represent pressure at water surface at time step $n+1$ and $n$, respectively. $\varphi$ is a weighing factor, which has been set to $0.5 . w_{I, T}$ is the vertical velocity at the top layer located at a distance of $0.25 \Delta z_{I}$ from the surface, which has been calculated by Eq. (27):

$w_{I, T}=0.25 w_{I, K_{\max }-1 / 2}+0.75 w_{I, K_{\max }+1 / 2}$

Substituting $\eta_{I}^{n+1}$ from Eq. (25), $w_{I, K_{\max }-1 / 2}^{n+1}$ from the momentum equation in $z$-direction, and $w_{I, K_{\max }+1 / 2}^{n+1}$ from the column integration equation of continuity (Eqs. 9 and 10) into Eq. (26), the pressure equation for the top layer is obtained.
The pressure equations can then be summarized for the whole domain as follows:

$$
\overline{\bar{A}}_{I} \bar{P}_{I-1}^{* n+1}+\overline{\bar{B}}_{I} \bar{P}_{I}^{* n+1}+\overline{\bar{C}}_{I} \bar{P}_{I+1}^{* n+1}=\bar{D}_{I}
$$

This comprises a block tri-diagonal matrix in the form of Eq. (29), which is an $\left(I_{\max }\right) \times\left(I_{\max }\right)$ matrix, where $I_{\max }$ is the number of columns. Each block of the block tri-diagonal matrix takes the form of a $\left(K_{\max }\right) \times\left(K_{\max }\right)$ matrix, where $K_{\max }$ is the number of rows. Hence, the pressure coefficient in each cell is correlated to the two upper layers and two lower layers of the cell together with the layer to which the cell belongs except at the last row where all pressures of the corresponding column are contained.

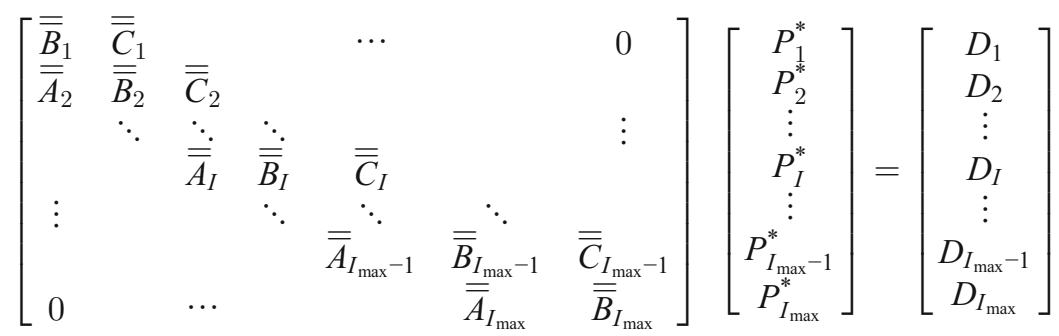

The block tri-diagonal matrix was solved by block forward elimination and back-substitution (Twizell 1984 and Golub and Van Loan 1989). The matrices are diagonally dominated; hence, no pivoting is required (Kincaid and Cheney 1991).

Having calculated the pressure values, the velocities in the domain are then computed as follows:

$\frac{V^{n+1}-V^{*}}{\Delta t}+\nabla P^{* n+1}=0$

The velocities on the free surface are then calculated by solving the dynamic free surface boundary condition and the continuity equation simultaneously. Water elevation is computed through the solution of free surface equation obtained from the application of normal and tangential dynamic boundary conditions and the integration of the continuity equation over total depth with the application of kinematic conditions at rigid bed and free surface. Normal and tangential boundary conditions have been applied at the water and fluid mud interface to keep the consistency of solution in the two-layer system. The interface elevation is obtained by the application of the integration of continuity equation over mud depth and kinematic boundary conditions at bed and interface. Gridding is then updated in mud and water layers independently. In the mud layer, grid geometry is computed and updated according to the interface and bed levels, and in the water layer, the grid is updated according to the interface and free surface levels.

\section{Model validations}

To validate the new improved model, three hydrodynamic tests of free surface flow problems with significant nonhydrostatic pressure distribution have been chosen. Predicted values for small-amplitude progressive wave train in deep water and solitary wave propagation in a constant water depth have been compared with the corresponding analytical solutions for water elevation, pressure field, and velocity distribution. The simulated values of free surface elevation for nonlinear short wave propagation in a constant water depth have been compared against the measured values reported in the literature. A two-layer system in which a layer of clear water overlies a thin layer of viscous mud was considered to simulate wave-mud interaction. The predicted values of wave 
height have been compared versus measured values. The wave damping coefficients have also been compared with the theoretical- and experimental-based values for alternative wave heights and frequencies. The ratio of the interfacial to surface wave amplitudes has been computed and compared with theoretical- and experimental-based data. The variation of surface wave number values against mud layer thickness has also been modeled and compared with analytical solution results.

\subsection{Hydrodynamic tests}

\subsubsection{Small-amplitude progressive wave train in deep water}

A wave train produced by a flap-type wavemaker with the assumption of an inviscid flow was simulated. The wave period was taken to be equal to $T=5 \mathrm{~s}$ and the wave height to $H=0.5 \mathrm{~m}$. The water depth was set to $d=15 \mathrm{~m}$, giving a wave number equal to $k \approx 0.16344$. According to Eq. (17), the sinusoidal velocity of the wave maker at the water surface was set to $0.2604 \mathrm{~m} / \mathrm{s}$. The velocity for different layers is a linear function being zero at the bed and equal to the maximum velocity at the still water surface. This constituted the left-hand side boundary condition of the domain, with the Neumann boundary condition being set to zero for $w$ in the $x$ direction. For the right-hand side, a free exit for water was maintained by setting a zero dynamic pressure condition at the far end of the domain. The Dirichlet boundary condition was set to zero for $w$-velocities at the bed (i.e., a flat bed), and a Neumann boundary condition equal to zero was prescribed for $u$ in the $z$-direction. The domain was considered to be $1,500 \mathrm{~m}$ in length, which was discretized by grids equal to $\Delta x=1 \mathrm{~m}$ in the $x$-direction. The depth of the domain was divided into 15 layers. Time step was set at $\Delta t=0.05 \mathrm{~s}$. Using an analytical solution, the celerity of the wave was calculated to be $7.692 \mathrm{~m} / \mathrm{s}$.

Comparisons of the numerical simulations and the analytical solutions were undertaken after 28 periods, corresponding to $t=140 \mathrm{~s}$. Equation (31) demonstrates the relevant surface water elevation, horizontal and vertical velocity components, and dynamic pressure under the second-order Stokes wave train (Dean and Dalrymple 1991):

$$
\begin{aligned}
\Theta= & k x-\omega t \\
\eta= & \frac{H}{2} \cos \Theta+\frac{\pi H^{2}}{8 L} \frac{\cosh k d}{\sinh ^{3} k d}[2+\cosh 2 k d] \cos 2 \Theta \\
u= & \frac{\pi H}{T} \frac{\cosh k z}{\sinh k d} \cos \Theta+\frac{3}{4}\left(\frac{\pi H}{L}\right) \frac{\pi H}{T} \frac{\cosh 2 k z}{\sinh ^{4} k d} \cos 2 \Theta \\
w= & \frac{\pi H}{T} \frac{\sinh k z}{\sinh k d} \sin \Theta+\frac{3}{4}\left(\frac{\pi H}{L}\right) \frac{\pi H}{T} \frac{\sinh ^{2 k z}}{\sinh ^{4} k d} \sin 2 \Theta \\
P^{*}= & g \frac{H}{2} \frac{\cosh k z}{\cosh k d} \cos \Theta+\frac{3}{4} g \frac{\pi H^{2}}{L} \frac{1}{\sinh 2 k d} \times\left[\frac{\cosh 2 k z}{\sinh ^{2} k d}-\frac{1}{3}\right] \cos 2 \Theta \\
& -\frac{1}{4} g \frac{\pi H^{2}}{L} \frac{1}{\sinh 2 k d} \times[\cosh 2 k z-1]
\end{aligned}
$$

where $L$ is the wave length. Simulated free surface water elevations have been plotted and compared against an analytical solution in Fig. 3. Despite small discrepancies, the wave does not dissipate or decay over time, and the difference does not grow. The corresponding dynamic pressure and velocity field are shown in Fig. 4, confirming the capability of the numerical model to predict the progressive waves.

\subsubsection{Solitary wave propagation in a constant water depth}

Propagation of solitary wave in a constant water depth is performed to evaluate the capability of the model in simulating non-linear terms. According to the potential flow theory, a small-amplitude solitary wave propagates at a constant speed without changes in form, amplitude, and velocities in a constant depth (Mei 1983). The same wave condition described by Yuan and $\mathrm{Wu}$ (2004) is prescribed. A solitary wave with an amplitude of $1 \mathrm{~m}$ propagates in a constant water depth of $10 \mathrm{~m}$. At the inlet, the time series of horizontal velocities based on the analytical solution of Sorensen (1997) was applied, where the initial position of a wave crest was specified at $x=-150 \mathrm{~m}$. Outlet and bed boundary conditions were similar to those which have been used for the progressive wave test. The domain was considered to be $2,000 \mathrm{~m}$ in length,
Fig. 3 Comparisons of free surface elevation between analytical solution and numerical simulation results under Stokes wave train at $t=140 \mathrm{~s}$

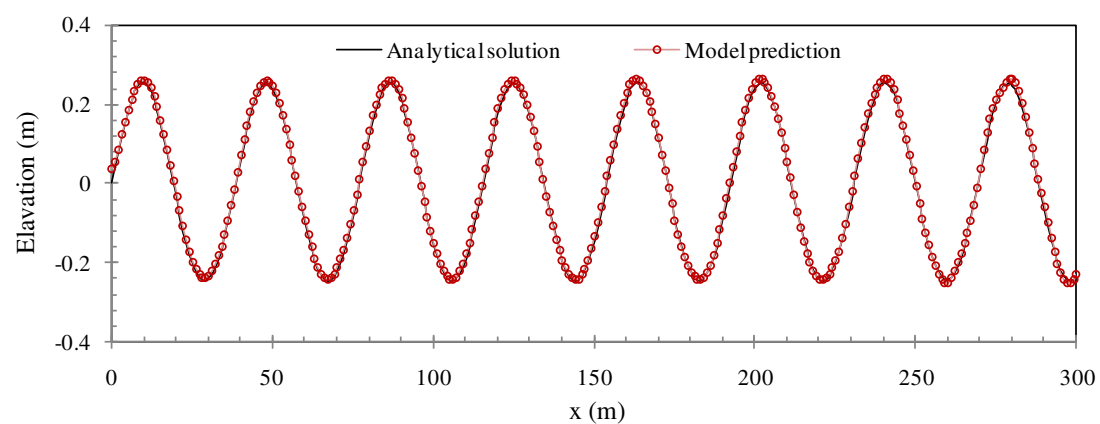


a

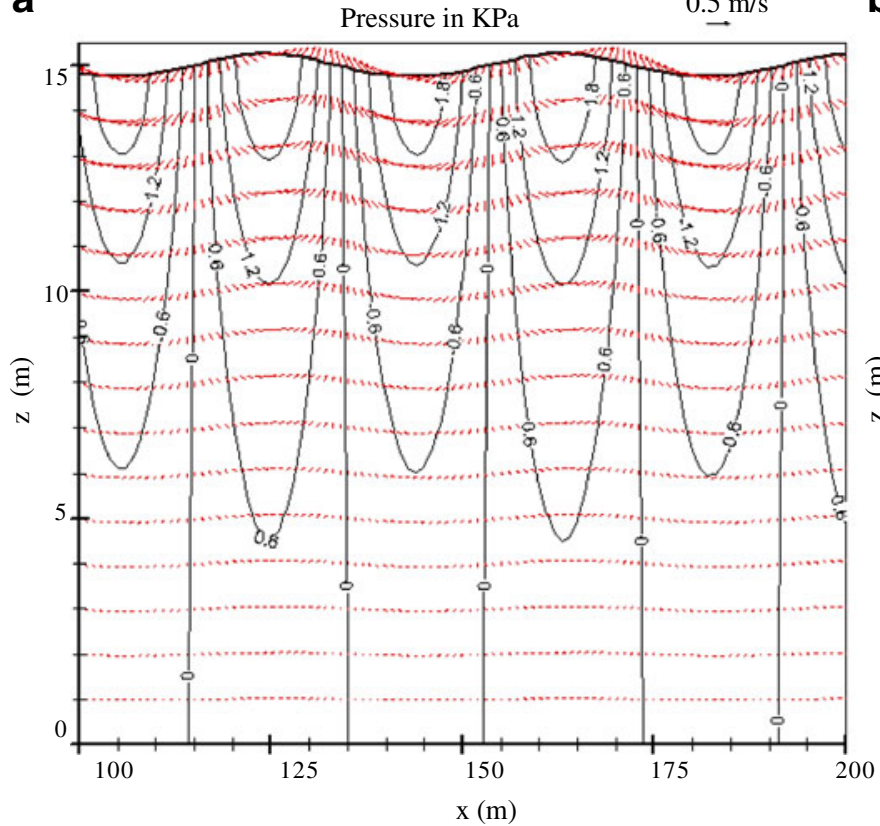

b

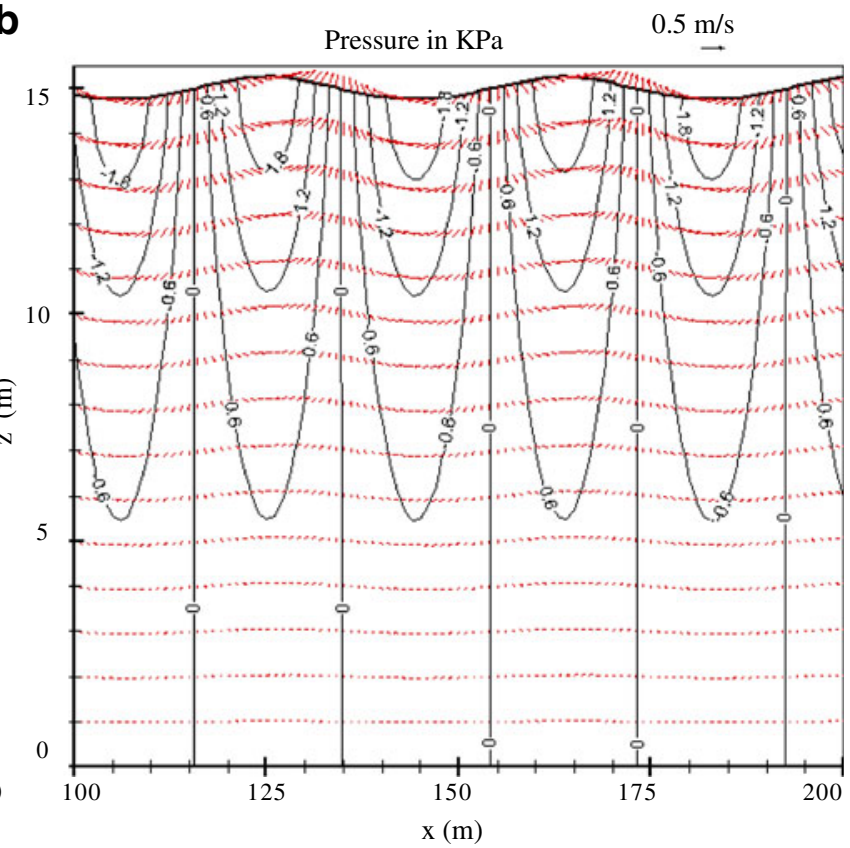

Fig. 4 Comparison of dynamic pressure and velocity fields under Stokes wave train at $t=140 \mathrm{~s}$. a Model prediction. b Analytical solution

which was discretized by grids equal to $\Delta x=2 \mathrm{~m}$ in the $x$ direction. The depth of the domain was divided into ten layers. Time step was set to $\Delta t=0.1 \mathrm{~s}$ and the wave celerity and Courant number were calculated to be $c=10.388 \mathrm{~m} / \mathrm{s}$ and $C r=$ 0.519 , respectively.

Comparisons of the numerical prediction and analytical solution for free surface elevation at $t=45,90$, 135, and $180 \mathrm{~s}$ are shown in Fig. 5. The dynamic pressure and velocity field at $t=180 \mathrm{~s}$ are shown in Fig. 6. The horizontal and vertical velocities at the free surface are also shown and compared with analytical results in Fig. 7. Overall, numerical predictions are almost identical to the analytical solutions, suggesting the capability of the model in simulating non-linear terms, i.e., advection, in NavierStokes equations.

\subsubsection{Non-linear short wave propagation in a constant water depth}

Propagation of a non-linear sinusoidal short wave in intermediate water depth has been simulated. The water elevation and non-linear behavior predicted by the model have been compared with measurements reported by Chapalain et al. (1992). The experiments were conducted in a 35.54-m-long, 0.4-m-deep wave flume, and the initial water elevation was set to zero. The incident wave height of $H=0.084 \mathrm{~m}$ and the wave period of $T=2.5 \mathrm{~s}$ were adapted, and the computational domain of $70 \mathrm{~m}$ in length has been considered. The numerical domain was discretized by grids equal to $\Delta x=0.1 \mathrm{~m}$ in the $x$-direction. The depth of the domain was divided into ten layers and the time step was set to $\Delta t=0.01 \mathrm{~s}$. Comparisons
Fig. 5 Comparisons of free surface elevation between analytical solution and numerical simulation results for solitary wave propagation in a constant water depth at $t=45,90,135$, and $180 \mathrm{~s}$

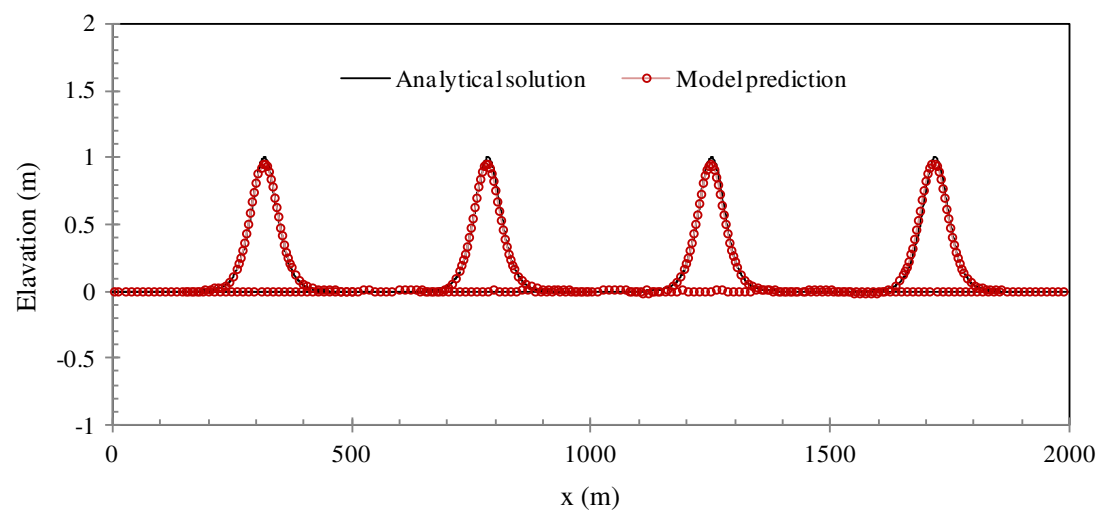



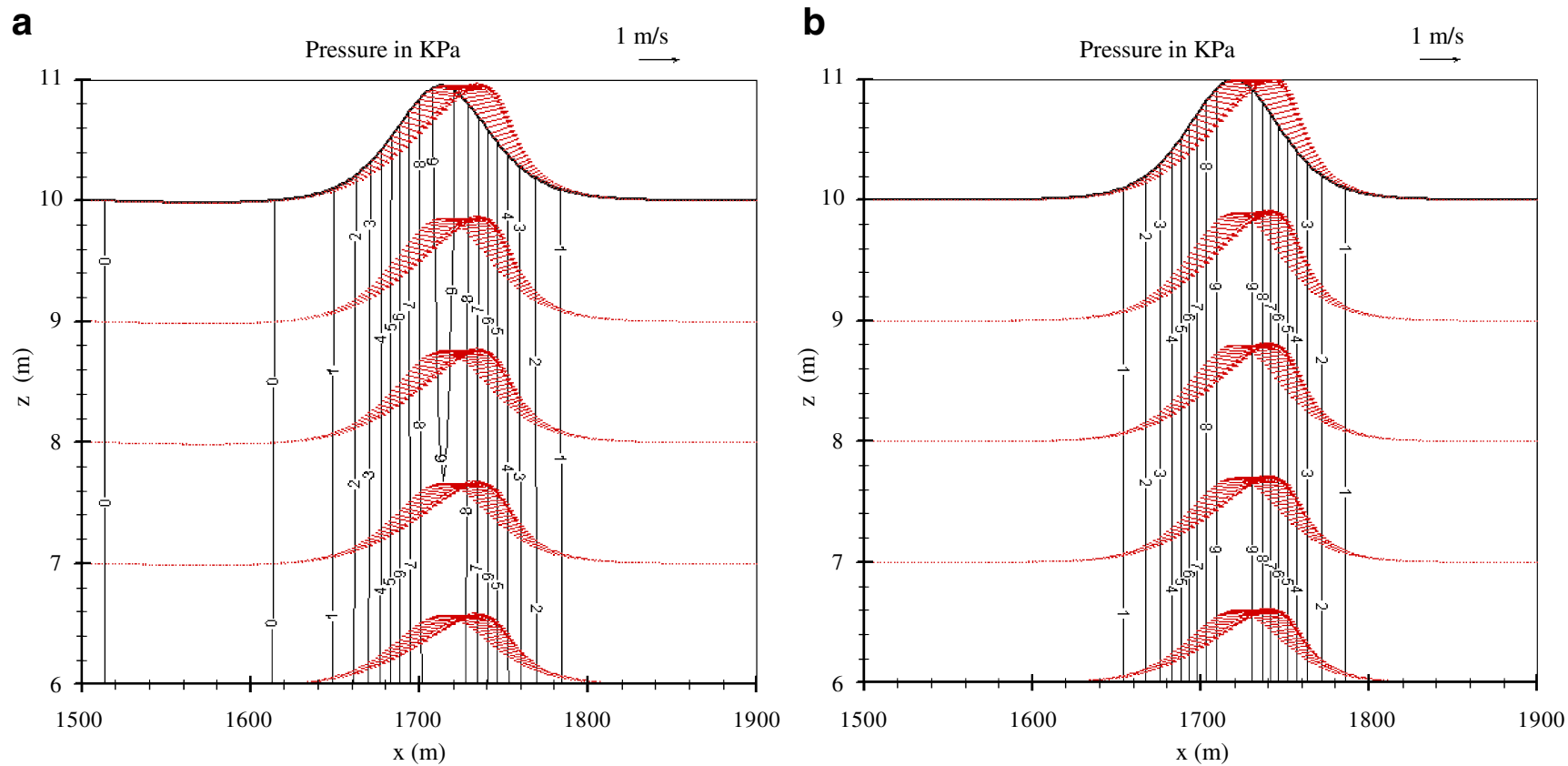

Fig. 6 Comparison of dynamic pressure and velocity field for solitary wave propagation in a constant water depth at $t=180 \mathrm{~s}$. a Model prediction. b Analytical solution

of the predicted free surface elevations with laboratory measurements are shown in Fig. 8 at five points in the longitudinal direction of the flume and for 30 seconds. The numerical results show a good agreement and reasonable accuracy, confirming the model's ability in the prediction of non-linear behavior of short waves.
Fig. 7 Predicted horizontal and vertical velocity components compared with analytical solution for solitary wave propagation in a constant water depth at $\mathrm{t}=45,90,135$, and 180 s. a Horizontal velocity components. b Vertical velocity components

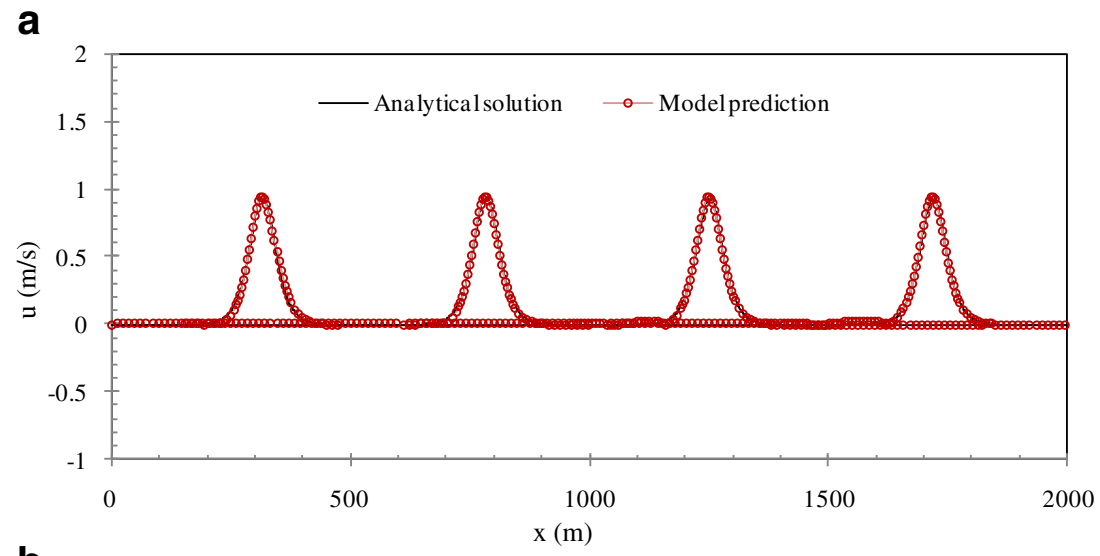

b

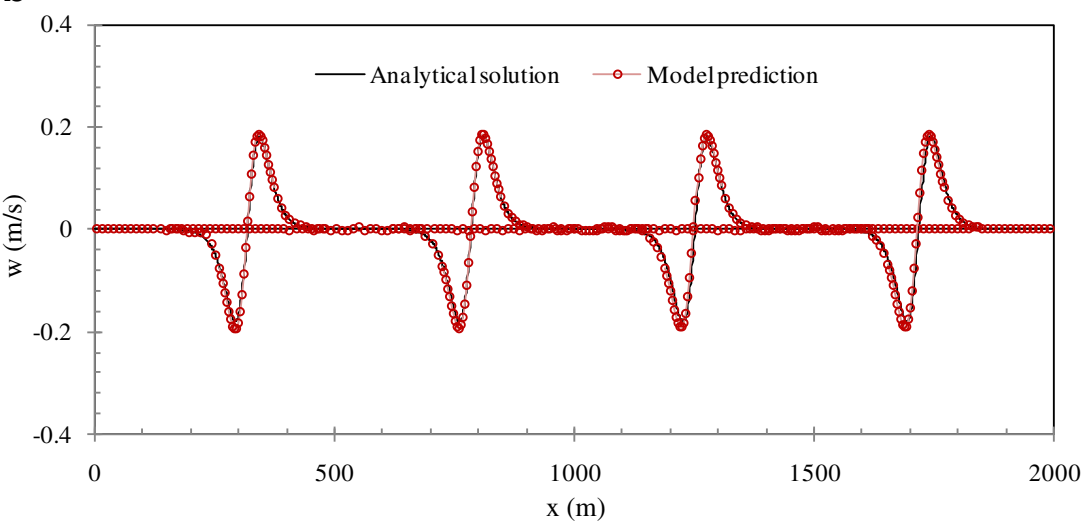


Fig. 8 Comparisons of freesurface elevation between experimental data and numerical predictions for non-linear sinusoidal wave propagation along the wave flume
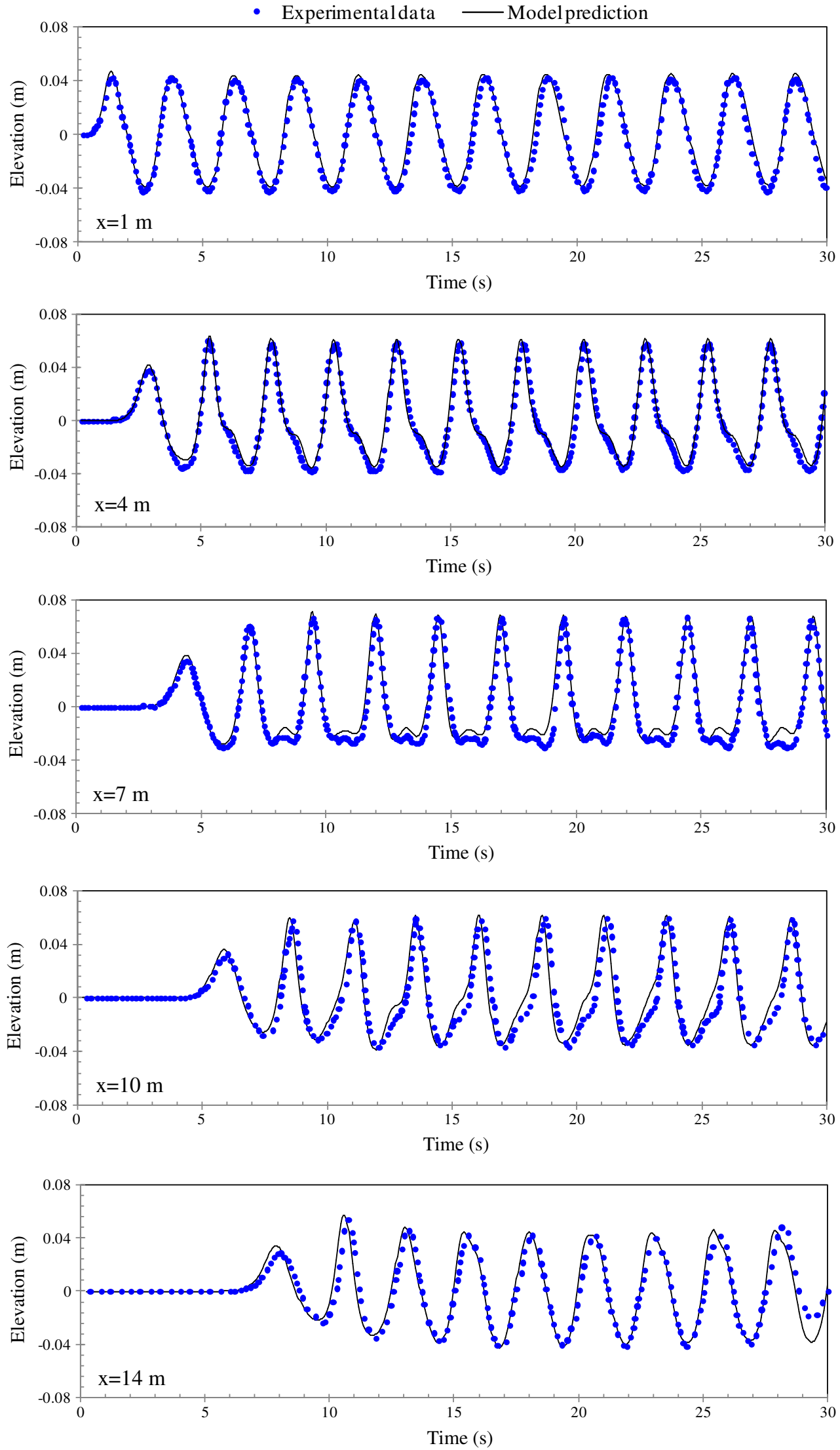


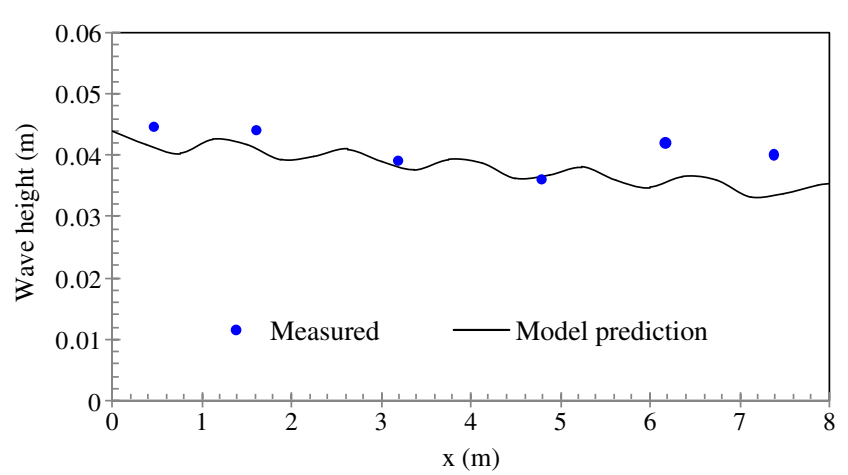

Fig. 9 Comparisons between measured values (De Wit (1995)) versus numerical predictions of wave height for sinusoidal wave propagation along the wave flume in a system of water and fluid mud

\subsection{Wave-mud interaction tests}

A two-layer system in which a layer of clear water overlies a thin layer of viscous mud was considered to predict wavemud interaction. The simulated results have been compared with the laboratory measurements reported by De Wit (1995) and Sakakiyama and Bijker (1989). The flume used by De Wit (1995) was $40 \mathrm{~m}$ in length, with a width and depth of $0.8 \mathrm{~m}$. The flume was fitted with a false floor to accommodate the mud layer with a length of $8 \mathrm{~m}$. The initial mud and water depth were 0.115 and $0.325 \mathrm{~m}$, respectively. The mud viscosity was measured to be $2.7 \times 10^{-3} \mathrm{~m}^{2} / \mathrm{s}$ and for water it was $1.3 \times 10^{-6} \mathrm{~m}^{2} / \mathrm{s}$. Numerical parameters were set, $\Delta x=$ $0.03 \mathrm{~m}$ and $\Delta t=0.005 \mathrm{~s}$, and the simulation time was $55 \mathrm{~s}$. Water depth was divided into 15 layers, and mud thickness was divided into six layers.

The laboratory experiments of Sakakiyama and Bijker (1989) were performed in a wave flume of $24.5 \mathrm{~m}$ in length,
$0.50 \mathrm{~m}$ in width, and $0.57 \mathrm{~m}$ in depth at the Laboratory of Fluid Mechanics, Delft University of Technology. The mixture of commercial kaolinite and water was applied as mud layer of $12 \mathrm{~m}$ in length. The initial thickness of the mud layer was $0.09 \mathrm{~m}$, and water depth was fixed at $0.30 \mathrm{~m}$. Surface wave heights were measured every half meter along the wave flume by using two capacitance-type wave gauges. Although mud behaves as a non-Newtonian fluid, an experimentalbased relationship between the apparent kinematic viscosity and the mud density has been obtained and reported by Sakakiyama and Bijker (1989) for simplicity and has been used for the values of viscosity in the simulations. The numerical geometry was taken to be the same as the laboratory setup. The numerical domain was discretized by grids of $\Delta x=0.1 \mathrm{~m}$ in the flow direction. Water depth was divided into ten layers, and mud thickness was divided into three layers, resulting in a total number of 13 layers in the $z$ direction. Time step was set to $0.0025 \mathrm{~s}$, and the simulation time was $70 \mathrm{~s}$.

\subsubsection{Surface wave propagation}

De Wit's results for the experiment, in which China clay was used, have been selected for the comparison of surface wave propagation over the mud layer, and the values of wave height variations over mud layer have only been considered. The density of mud was $1,300 \mathrm{~kg} / \mathrm{m}^{3}$, and the water density was $1,000 \mathrm{~kg} / \mathrm{m}^{3}$. The incident wave height of $H_{0}=0.045 \mathrm{~m}$ and the wave period of $T=1.5 \mathrm{~s}$ were applied.

Figure 9 shows the measured values of variation of wave height at six locations along the flume in comparison with model-predicted values. In spite of the existence of a general
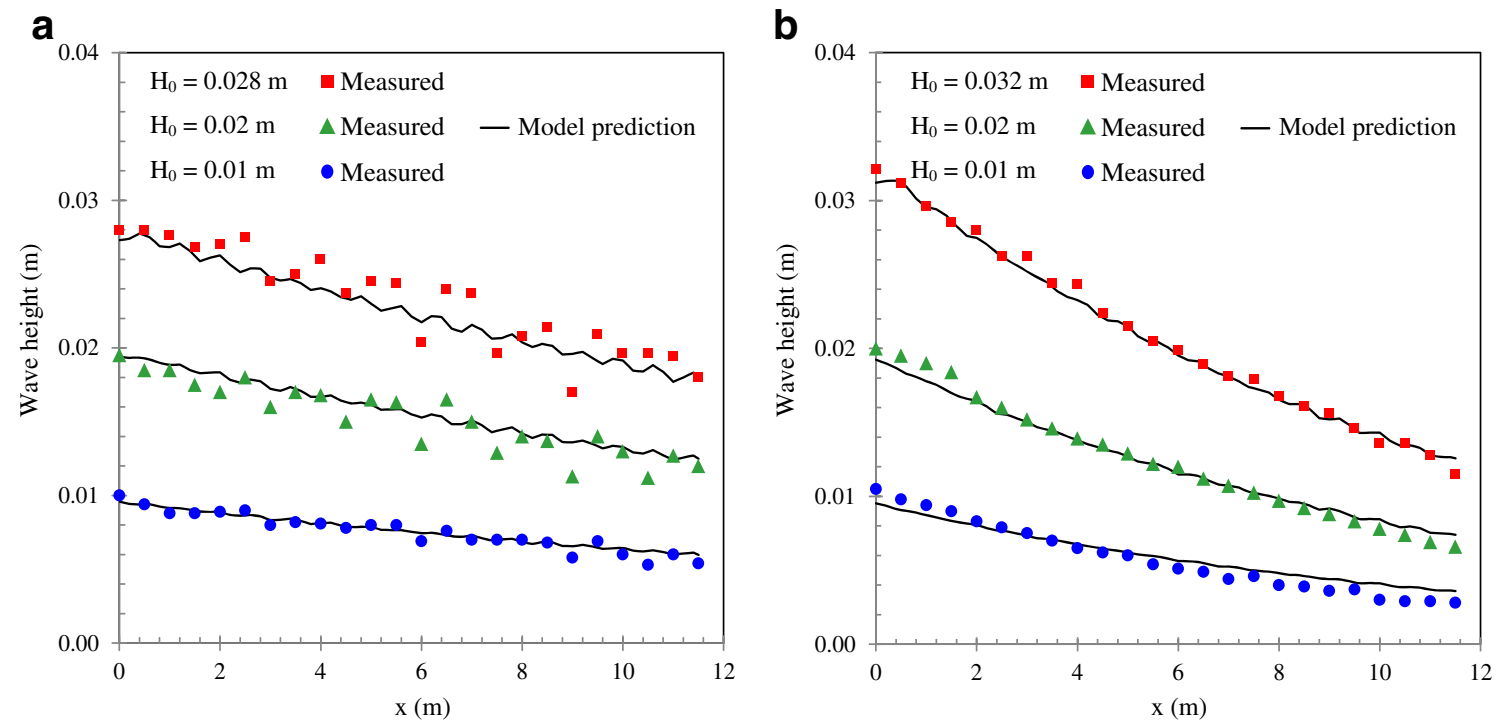

Fig. 10 Comparisons of wave height between measured values and numerical predictions for sinusoidal wave propagation along the wave flume in a system of water and fluid mud. a Laboratory set "a". b Laboratory set "b" 
Table 1 Measured and predicted values of wave height for sinusoidal wave propagation along the wave flume in a system of water and fluid mud. $H_{0}, H_{\mathrm{M}}$ (measured wave height), and $H_{\mathrm{P}}$ (predicted wave height) are in millimeters

\begin{tabular}{|c|c|c|c|c|c|c|c|c|c|c|c|c|}
\hline \multirow{3}{*}{$\begin{array}{l}x \\
(\mathrm{~m})\end{array}$} & \multicolumn{6}{|c|}{ Laboratory data set "a" } & \multicolumn{6}{|c|}{ Laboratory data set "b" } \\
\hline & \multicolumn{2}{|c|}{$H_{0}=10$} & \multicolumn{2}{|c|}{$H_{0}=20$} & \multicolumn{2}{|c|}{$H_{0}=28$} & \multicolumn{2}{|c|}{$H_{0}=10$} & \multicolumn{2}{|c|}{$H_{0}=20$} & \multicolumn{2}{|c|}{$H_{0}=32$} \\
\hline & $H_{\mathrm{M}}$ & $H_{\mathrm{P}}$ & $H_{\mathrm{M}}$ & $H_{\mathrm{P}}$ & $H_{\mathrm{M}}$ & $H_{\mathrm{P}}$ & $H_{\mathrm{M}}$ & $H_{\mathrm{P}}$ & $H_{\mathrm{M}}$ & $H_{\mathrm{P}}$ & $H_{\mathrm{M}}$ & $H_{\mathrm{P}}$ \\
\hline 0 & 10.0 & 9.59 & 19.50 & 19.40 & 28.00 & 27.30 & 10.5 & 9.53 & 20.00 & 19.25 & 32.10 & 31.18 \\
\hline 1 & 8.80 & 9.16 & 18.50 & 18.84 & 27.60 & 26.83 & 9.40 & 8.69 & 19.00 & 17.87 & 29.63 & 29.63 \\
\hline 2 & 8.90 & 8.88 & 17.00 & 18.35 & 27.00 & 26.27 & 8.30 & 8.01 & 16.70 & 16.53 & 28.00 & 27.51 \\
\hline 3 & 8.00 & 8.33 & 16.00 & 17.23 & 24.50 & 24.75 & 7.50 & 7.31 & 15.20 & 15.06 & 26.20 & 25.24 \\
\hline 4 & 8.10 & 8.12 & 16.80 & 16.66 & 26.00 & 24.05 & 6.50 & 6.69 & 13.90 & 13.86 & 24.30 & 23.30 \\
\hline 5 & 8.00 & 7.86 & 16.50 & 16.16 & 24.50 & 23.00 & 6.00 & 6.17 & 12.90 & 12.74 & 21.50 & 21.50 \\
\hline 6 & 6.90 & 7.43 & 13.50 & 15.29 & 20.40 & 21.75 & 5.10 & 5.62 & 12.00 & 11.55 & 19.85 & 19.50 \\
\hline 7 & 7.00 & 7.30 & 15.00 & 15.08 & 23.70 & 21.57 & 4.40 & 5.23 & 10.70 & 10.79 & 18.11 & 18.11 \\
\hline 8 & 7.00 & 6.85 & 14.00 & 14.19 & 20.80 & 20.37 & 4.00 & 4.79 & 9.70 & 9.88 & 16.77 & 16.55 \\
\hline 9 & 5.80 & 6.56 & 11.30 & 13.60 & 17.00 & 19.60 & 3.60 & 4.38 & 8.80 & 9.08 & 15.60 & 15.24 \\
\hline 10 & 6.00 & 6.45 & 13.00 & 13.30 & 19.60 & 19.15 & 3.00 & 4.10 & 7.80 & 8.48 & 13.60 & 14.29 \\
\hline 11 & 6.00 & 6.00 & 12.70 & 12.41 & 19.40 & 17.70 & 2.90 & 3.67 & 6.90 & 7.57 & 12.80 & 12.81 \\
\hline
\end{tabular}

trend of wave damping along the mud layer, the variation of the wave height is likely to be due to the ratio of wave height to water depth.

Characteristics of two set of laboratory experiments conducted by Sakakiyama and Bijker (1989) have been chosen for the comparison of wave damping coefficients for various initial wave heights. The laboratory measurement sets "a" and "b" have densities of 1,240 and $1,300 \mathrm{~kg} / \mathrm{m}^{3}$ for the mud layer respectively. The value of water density was taken as $\rho=$ $1,000 \mathrm{~kg} / \mathrm{m}^{3}$. At the inlet sinusoidal waves of period $T=1 \mathrm{~s}$ and alternative heights of $H_{0}=0.01,0.02$, and $0.028 \mathrm{~m}$ for simulating laboratory set "a" and $H_{0}=0.01,0.02$, and $0.032 \mathrm{~m}$ for simulating laboratory set "b" were initiated in an undisturbed two-layer system. Other boundary conditions were similar to the tests explained earlier. The numerical parameters are those explained in "Section 4.2". Calibration was made by adjusting the value of kinematic viscosity for the laboratory measurement set "a".

Figure 10 shows the wave heights along the wave flume for various initial wave heights for the laboratory sets "a" and " $b$ ". The comparisons made with the laboratory measurements show good agreements, while the predictions of waves with smaller amplitudes have less discrepancy with measured values for the laboratory set "a", and the opposite a

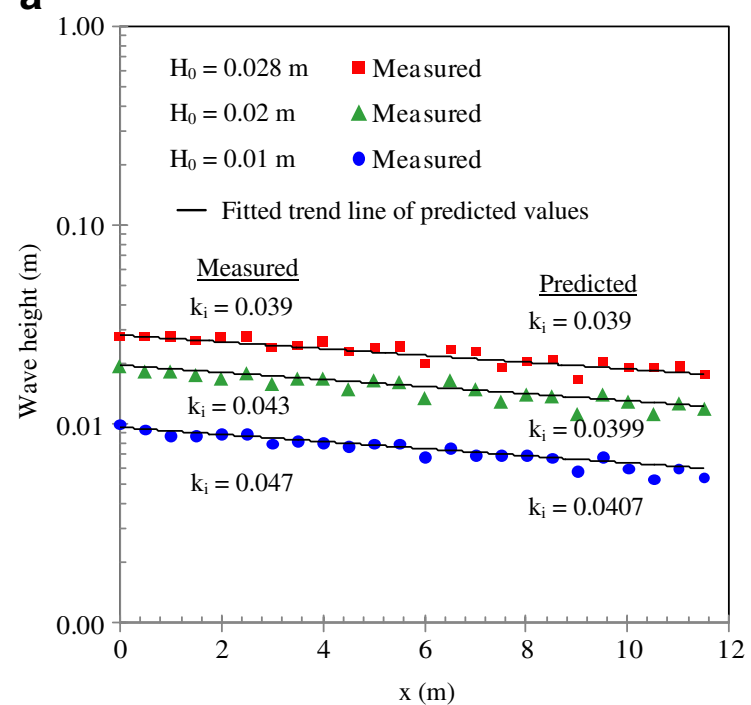

b

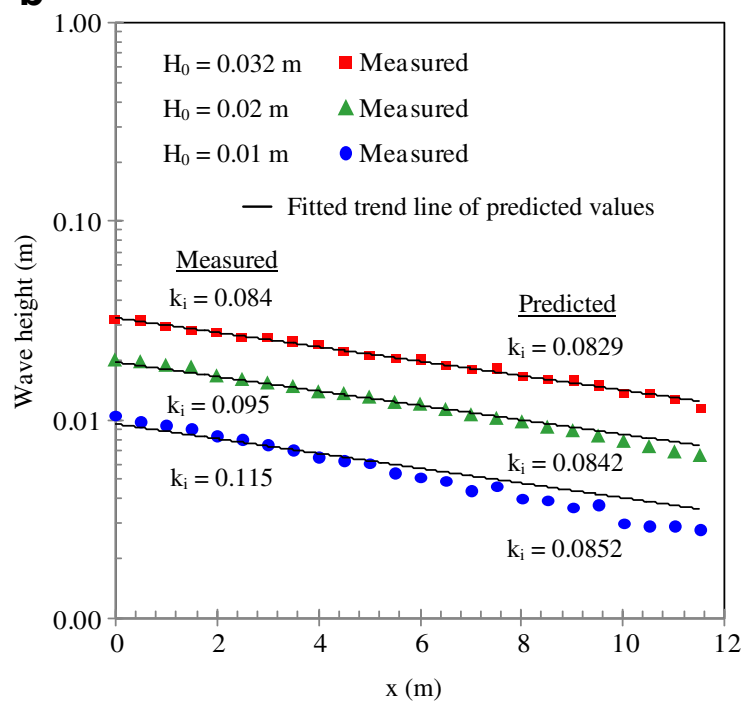

Fig. 11 Comparisons of wave damping coefficients and fitted trend lines of wave height of predicted results with measured values in a semi-logarithmic diagram for sinusoidal wave propagation along the wave flume in a system of water and fluid mud. a Laboratory set "a". b Laboratory set "b" 
is true for the laboratory set "b". The measured and predicted wave heights along the mud bed have also been summarized in Table 1 for the laboratory sets "a" and "b".

\subsubsection{Wave damping coefficient}

The wave damping coefficient $k_{i}$ is defined by Iwasaki and Sato (1972) as Eq. (32):

$$
H=H_{0} e^{-k_{i} x}
$$

where $H_{0}$ is the reference surface wave height at the origin, $H$ is either the predicted or measured wave height, and $x$ is the distance from the origin. The damping coefficients for measured and predicted values have been calculated and demonstrated for laboratory sets "a" and " $\mathrm{b}$ " and for various initial wave heights in Fig. 11. The fitted trend lines of predicted values of wave height in a semi-logarithmic diagram and the damping coefficients show better agreements with the corresponding measured values for larger amplitudes for both laboratory data sets "a" and "b".

The damping coefficients of measured and predicted values have also been calculated and plotted for both laboratory data sets for various wave frequencies in Fig. 12. The damping coefficients based on the theoretical values of Dalrymple and Liu (1978) and the theoretical solution of An (1993) have also been demonstrated in the graph. Dalrymple and Liu (1978) developed a theory for linear water wave propagation in a two-layer viscous fluid system, which gives the orbital motions in both the upper and lower layers. The solutions to the linearized Navier-Stokes

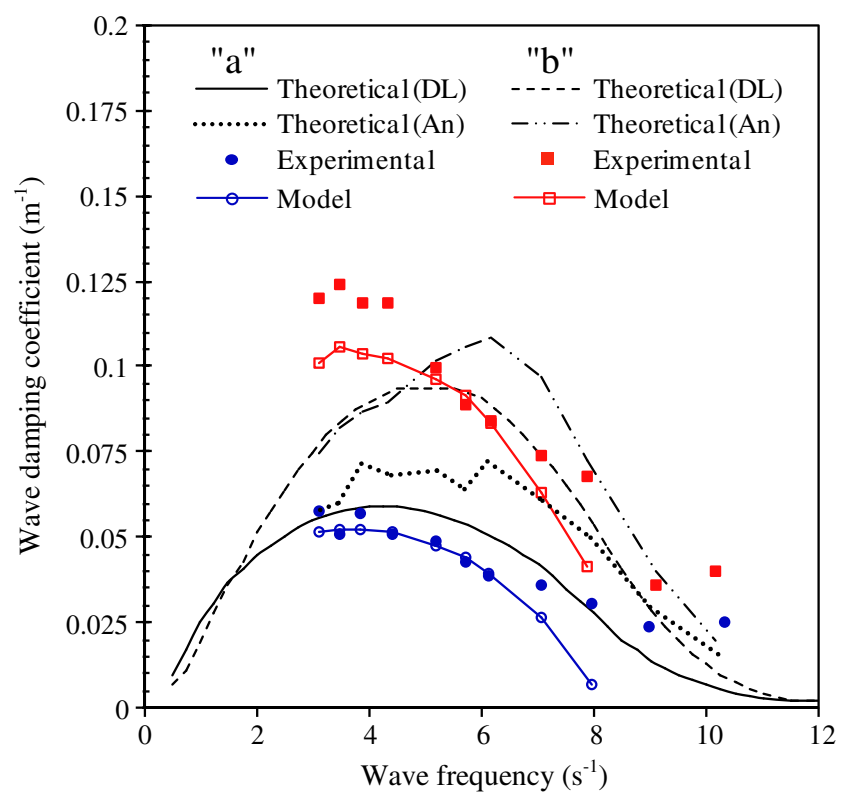

Fig. 12 Comparisons of wave damping coefficient versus wave frequency between measured, theoretical and predicted values. $D L$ Dalrymple and Liu (1978), An An (1993) equations are assumed to be separable and to be periodic in time and $x$-direction. An (1993) extended this theory by adding the visco-elastic-plastic model to present the rheological properties of mud. The numerical parameters are the same, with the exception of $\Delta x$, the value of which has been chosen such that the ratio of grid number per wave length varies between 14 and 18. The damping coefficients based on numerical simulation have better agreements with experimental-based calculations than both of the theoretical-based values for lower frequencies. However, for higher frequencies the predictions show a closer agreement with the theoretical values of Dalrymple and Liu (1978). The experimental-, theoretical-, and predicted-based values of the damping coefficients have also been tabulated in Table 2 for both laboratory data sets.

\subsubsection{Interfacial wave amplitude}

Figure 13 shows the computed ratio of the interfacial to surface wave amplitude for various wave frequencies for both laboratory sets "a" and "b" calculated by Sakakiyama and Bijker (1989). The corresponding experimental results and the analytical solution of Dalrymple and Liu (1978) have also been graphed for comparison. For both laboratory data sets, the simulated values are between the experimental-based values and the theoretical solution. The experimental-, theoreticaland numerical-based values of the ratio of the interfacial to surface wave amplitude have also been presented in Table 3 for both laboratory sets.

\subsubsection{Wave number}

The variation of dimensionless surface wave number values versus dimensionless mud layer thickness is plotted in Fig. 14. The agreement with the results of the theoretical solution of Dalrymple and Liu (1978) is quite good.

\section{Discussion}

Fluid mud, in which the particles are largely fluid-supported, may result from rapid deposition or liquefaction of a mud bed due to wave action. Compliant or fluid mud can oscillate as waves pass over and cause wave heights to attenuate significantly. In addition to upward entrainment or downward movement due to dewatering, it is easily displaced under the effect of external forces, e.g., pressure gradients at the lutocline or gravity on inclined beds. Water waves propagating over a fluid mud bed are attenuated mainly due to energy dissipation in the fluid mud layer. The attenuation of wave height on a horizontal bed is usually approximated by an exponential function, which follows from the standard harmonic solution that satisfies the equation of motion for a progressive sinusoidal wave. 


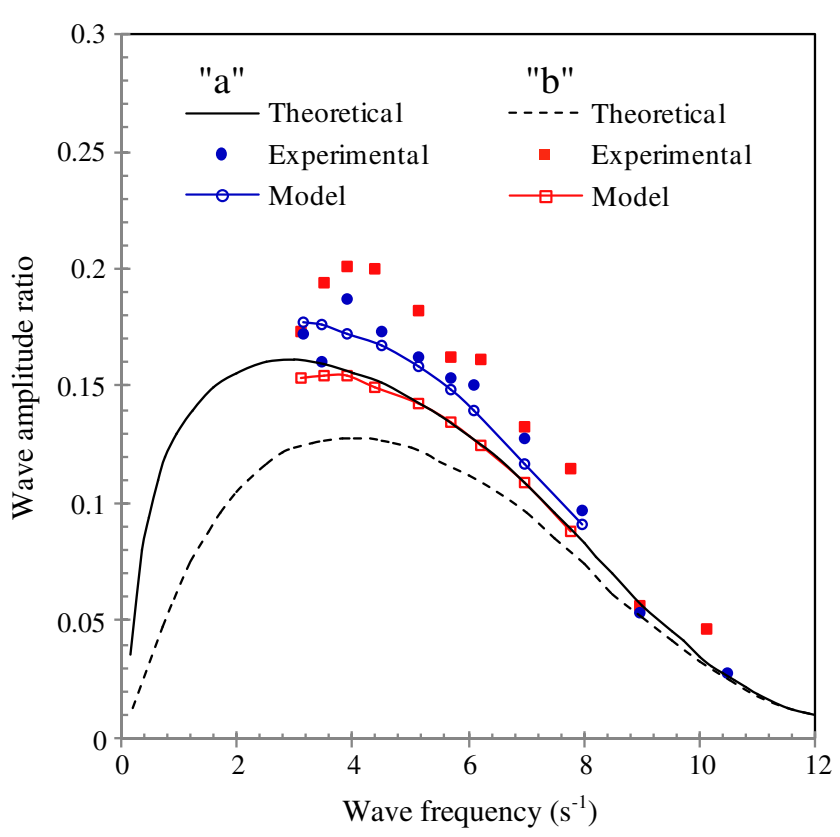

Fig. 13 Comparisons of the ratio of the interfacial to surface wave amplitude versus wave frequency between experimental-based values of Sakakiyama and Bijker (1989), theoretical results of Dalrymple and Liu (1978), and simulated results

In the present numerical formulation, two distinct processes of bed fluidization and surface erosion are not considered. It has been assumed that the mud is fluidized at the beginning of the simulation and does not change throughout, and the viscosity has been taken to be constant. However, the fluid mud depth and thickness may vary throughout the simulation, and the solution is updated accordingly.

Suitable rheological models of mud should be adopted in order to investigate wave-mud interaction. Mud, in general, may range from being a highly rigid and weakly viscous material to one that can be approximated as a purely viscous fluid, depending on the properties of the constituent sediment and the ambient fluid. In the present formulation, mud bed is treated as a Newtonian fluid which has greater viscosity and higher density than water. This simple treatment enables using Navier-Stokes equations for the two-layer fluid system of mud and water. A higher number of the rheological parameters should be determined in order to apply more sophisticated rheological models, e.g., viscoelastic, visco-plastic or viscoelastic-plastic. The problems associated with measuring these parameters affect the accuracy of simulating wave-mud interaction in a way that the use of non-Newtonian rheological models in practical applications may not necessarily lead to an increase of the accuracy of predictions.

For hydrodynamic tests, three free surface flow problems have been simulated. Simulation of small-amplitude progressive wave in deep water shows excellent agreements with the analytical solution of second-order Stokes wave train, which confirms the capability of the numerical model for simulating progressive waves. Simulation of propagation of a solitary wave in a constant water depth as the second hydrodynamic test showed very close predictions to the analytical solutions. The small asymmetry of the pressure distribution under the solitary wave is due to the small asymmetry in the prediction of surface water elevation of the wave. The ability of the model in prediction of non-linear behavior of short waves was also confirmed by reasonable accuracy and good agreements obtained from the simulation of non-linear short wave propagation in a constant water depth as the third hydrodynamic test.

Wave-mud interaction has been simulated for a two-layer system consisting of clean water and underlying fluid mud. In comparison with the experimental data of De Wit (1995) in Fig. 9, the ratio of wave height to water depth $(\sim 0.14)$ may

Table 2 The experimental-, theoretical- and predicted-based damping coefficient values for various wave frequencies

\begin{tabular}{|c|c|c|c|c|c|c|c|c|c|}
\hline \multirow[t]{4}{*}{$T(\mathrm{~s})$} & \multicolumn{4}{|c|}{ Laboratory data set "a" } & \multirow[t]{4}{*}{$T(\mathrm{~s})$} & \multicolumn{4}{|c|}{ Laboratory data set "b" } \\
\hline & \multicolumn{4}{|l|}{$k_{\mathrm{i}}\left(\mathrm{m}^{-1}\right)$} & & \multicolumn{4}{|l|}{$k_{\mathrm{i}}\left(\mathrm{m}^{-1}\right)$} \\
\hline & \multirow[t]{2}{*}{ Experimental } & \multicolumn{2}{|c|}{ Theoretical } & \multirow[t]{2}{*}{ Numerical } & & \multirow[t]{2}{*}{ Experimental } & \multicolumn{2}{|c|}{ Theoretical } & \multirow[t]{2}{*}{ Numerical } \\
\hline & & DL & An & & & & DL & An & \\
\hline 2.01 & 0.058 & 0.056 & 0.058 & 0.052 & 2.02 & 0.121 & 0.077 & 0.075 & 0.101 \\
\hline 1.81 & 0.051 & 0.058 & 0.060 & 0.052 & 1.8 & 0.125 & 0.084 & 0.082 & 0.106 \\
\hline 1.63 & 0.057 & 0.059 & 0.072 & 0.052 & 1.62 & 0.120 & 0.089 & 0.087 & 0.104 \\
\hline 1.43 & 0.051 & 0.059 & 0.069 & 0.052 & 1.45 & 0.119 & 0.092 & 0.090 & 0.103 \\
\hline 1.21 & 0.047 & 0.057 & 0.070 & 0.048 & 1.21 & 0.102 & 0.094 & 0.102 & 0.097 \\
\hline 1.1 & 0.043 & 0.054 & 0.064 & 0.044 & 1.1 & 0.089 & 0.093 & 0.106 & 0.092 \\
\hline 1.03 & 0.039 & 0.051 & 0.073 & 0.040 & 1.02 & 0.084 & 0.089 & 0.109 & 0.084 \\
\hline 0.89 & 0.036 & 0.041 & 0.061 & 0.027 & 0.89 & 0.074 & 0.074 & 0.096 & 0.063 \\
\hline 0.79 & 0.031 & 0.029 & 0.050 & 0.007 & 0.8 & 0.068 & 0.058 & 0.073 & 0.041 \\
\hline
\end{tabular}

DL Dalrymple and Liu (1978), An An (1993) 
Table 3 The experimental-, theoretical-, and numerical-based values of the ratio of the interfacial to surface wave amplitude for various wave frequencies

\begin{tabular}{|c|c|c|c|c|c|c|c|}
\hline \multirow[t]{3}{*}{$T(\mathrm{~s})$} & \multicolumn{3}{|c|}{ Laboratory data set "a" } & \multirow[t]{3}{*}{$T(\mathrm{~s})$} & \multicolumn{3}{|c|}{ Laboratory data set "b" } \\
\hline & \multicolumn{3}{|c|}{ Wave amplitude ratio } & & \multicolumn{3}{|c|}{ Wave amplitude ratio } \\
\hline & Experimental & Theoretical & Numerical & & Experimental & Theoretical & Numerical \\
\hline 2.01 & 0.172 & 0.161 & 0.177 & 2.02 & 0.173 & 0.125 & 0.154 \\
\hline 1.81 & 0.160 & 0.160 & 0.176 & 1.8 & 0.194 & 0.127 & 0.155 \\
\hline 1.62 & 0.187 & 0.159 & 0.172 & 1.62 & 0.201 & 0.128 & 0.155 \\
\hline 1.4 & 0.173 & 0.152 & 0.167 & 1.43 & 0.200 & 0.127 & 0.150 \\
\hline 1.22 & 0.162 & 0.144 & 0.158 & 1.22 & 0.182 & 0.122 & 0.143 \\
\hline 1.1 & 0.154 & 0.135 & 0.149 & 1.1 & 0.162 & 0.116 & 0.135 \\
\hline 1.03 & 0.151 & 0.127 & 0.140 & 1.01 & 0.161 & 0.109 & 0.125 \\
\hline 0.9 & 0.128 & 0.108 & 0.117 & 0.9 & 0.133 & 0.096 & 0.109 \\
\hline 0.79 & 0.097 & 0.084 & 0.091 & 0.81 & 0.115 & 0.079 & 0.089 \\
\hline
\end{tabular}

have caused the variation of wave height-simulated values along the mud layer. The sudden increase in measured wave heights near the down wave end of the mud patch was likely to be caused by wave reflection from the back of the mud pit (Kaihatu et al. 2007), but the model does not simulate this effect.

The comparisons of wave height simulation against the laboratory measurements of Sakakiyama and Bijker (1989) show that for both cases "a" and "b", the predictions of the numerical model grow, moving from the upstream side of the mud section towards the downstream. For instance, for case "a" and for an initial wave height of $0.028 \mathrm{~m}$, the model underestimates the wave height for the upstream part of mud, while this underestimation lessens for the downstream part, and for case " $b$ " and for an initial wave height of $0.01 \mathrm{~m}$, the model underestimates the wave height for the upstream part of mud, while for the downstream part the predictions are overestimated. Larger discrepancies and steeper slopes may also be observed for the graphs of predicted values for laboratory data set "b", with a larger viscosity. Figure 15

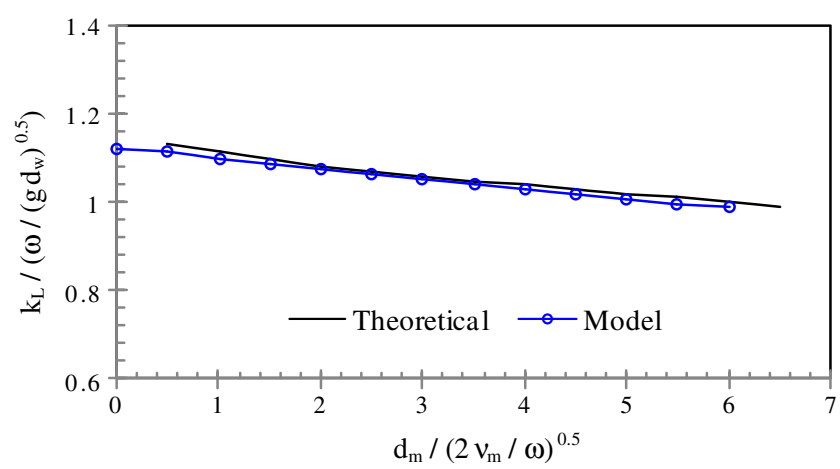

Fig. 14 Comparisons of dimensionless wave number versus dimensionless mud layer thickness between predicted and theoretical values of Dalrymple and Liu (1978) demonstrates this interpretation for both cases, which shows milder slopes for the graphs of simulated results compared with measured values. This may be explained by the fact that in the laboratory experiments, energy loss is due to both wave flume wall friction and bottom mud dissipation (Tsuruya et al. 1987); however, in the numerical simulations, no dissipation due to wall friction has been considered. The difference of the slopes of fitted graphs of model predictions and measured values grows for smaller wave heights. The comparison of predicted- and measured-based $k_{i}$ values confirm the same trend, which is shown in Fig. 16 for both cases "a" and "b", i.e., the agreement of numerical model predictions and measured values improves with the increase of the initial wave heights. Table 4 tabulates the percentage of relative error of predicted-based values compared to measured-based values of wave damping coefficients for various initial wave heights to quantify the discussion presented herein.

Regarding the relationship between the wave damping coefficient and wave frequency and according to Fig. 12, the closer graphs of the predicted- and measured-based values for low frequencies compared with the theoretical results may be influenced by the non-linear behavior of the wave propagation on the mud layer, whereas in the theory developed by Dalrymple and Liu (1978) and An (1993), linearized Navier-Stokes equations have been employed. This interpretation loses its documentary for high frequencies as it is guessed that wave propagation over the mud layer is more dominated by the non-Newtonian behavior of mud which is not included in the numerical model, and the nonlinear part of Navior-stokes equations, i.e., advection terms, is of less impact. The closer agreements of the theoretical results of An (1993) and the experimental values for high frequencies compared with low frequencies confirm this conclusion. Peak values are observed for both "a" and "b" laboratory-based data 
a

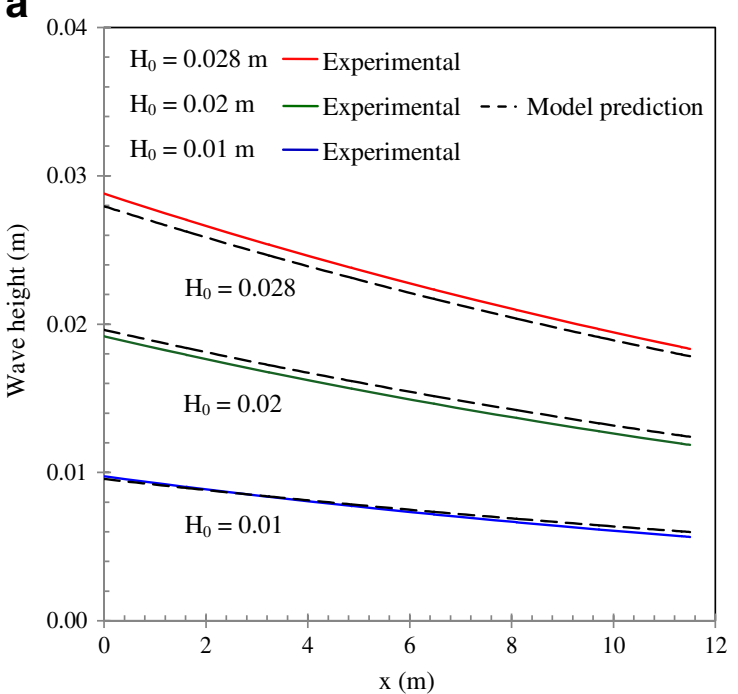

b

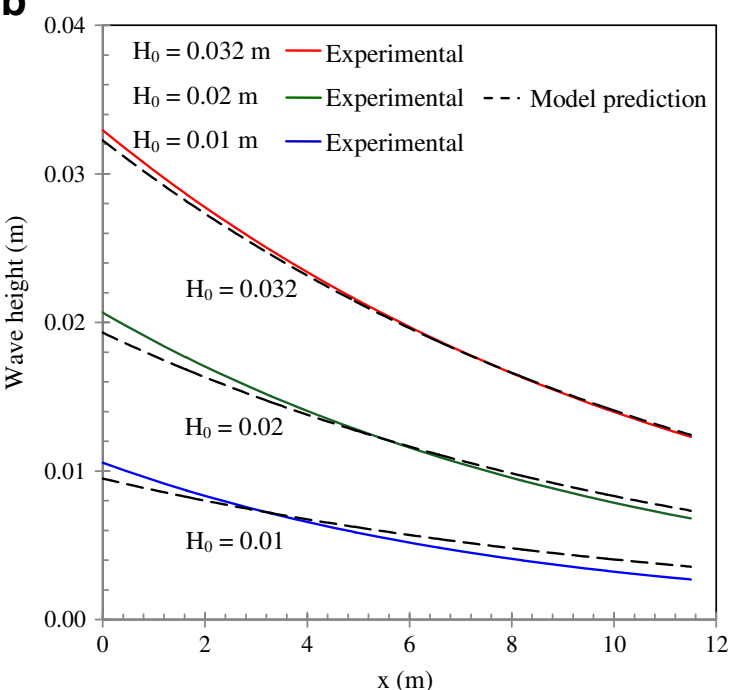

Fig. 15 Comparisons of fitted trend lines of wave height between experimental data and model predictions for sinusoidal wave propagation along the wave flume in a system of water and fluid mud. a Laboratory set "a". b Laboratory set " $b$ "

sets and relevant simulated-based values, which show small discrepancies for relevant frequencies.

The interfacial wave amplitude is influenced from the surface by the water wave and from the bottom by the viscosity and density of the mud. Figure 13 shows that the ratios of the interfacial to surface wave amplitude are larger for mud with a lower density according to the theoretical and numerical values. However, the experimental-based values show the reverse tendency, but this is an exception for mud with a density of $1,300 \mathrm{~kg} / \mathrm{m}^{3}$, and for the remaining tests of Sakakiyama and Bijker (1989), the same tendency is valid. For lower frequencies, with the rising effect of the surface waves on the mud layer the ratio increases; for further decreasing frequencies, however, the boundary layer thickness of the mud layer over the rigid bottom increases, and therefore with the increase of viscosity effect, the ratio decreases. This discussion may be observed from the numerical values of the ratio of the interfacial to surface wave amplitude which have a peak value for laboratory set "b". For laboratory set "a", however, no such peak is observed. In Fig. 14, with the increase of mud layer thickness, the wave number decreases, which is expected from shoaling effects.

Although the linearized Navier-Stokes equations may be theoretically solved using the approach of Dalrymple and Liu (1978) for the wave-mud interaction on horizontal bed, the proposed numerical model, using the non-linear NavierStokes equations and boundary conditions, is capable of computing the wave height transformation on other conditions, e.g., mud profiles and mud trenches, where analytical solutions do not exist. The new model may also be adjusted to simulate mild muddy slopes, and it is possible to extend the rheological model to non-Newtonian fluids.
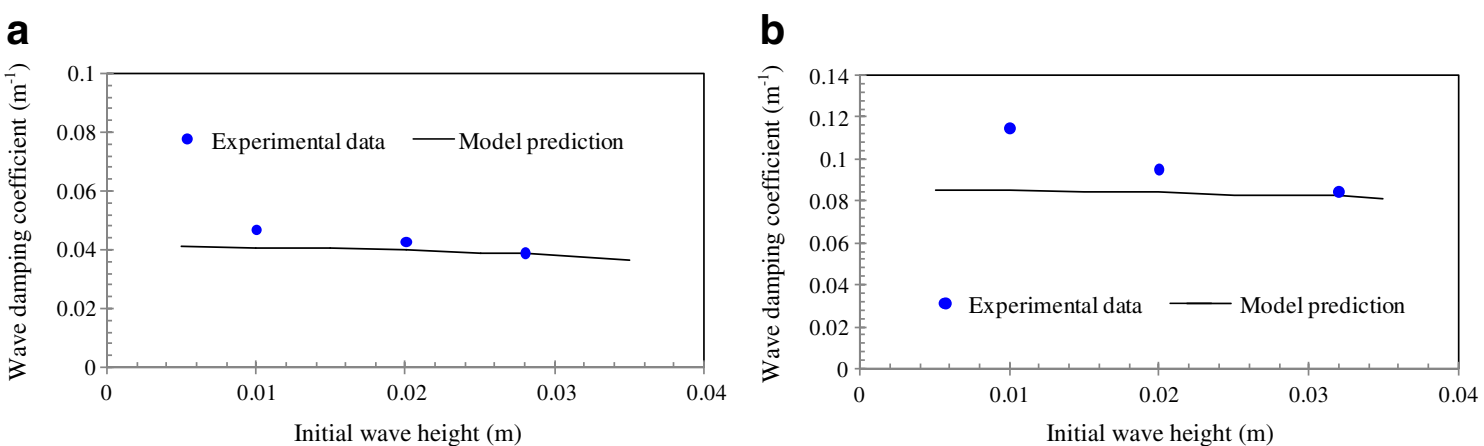

Fig. 16 Comparisons of wave damping coefficients based on experimental data and model predictions versus initial wave height. a Laboratory set "a". b Laboratory set "b" 
Table 4 Wave damping coefficient values derived from experimental data and model predictions and the corresponding relative error

\begin{tabular}{|c|c|c|c|c|c|c|}
\hline \multirow[t]{3}{*}{$k_{\mathrm{i}}\left(\mathrm{m}^{-1}\right)$} & \multicolumn{3}{|c|}{ Laboratory data set "a" } & \multicolumn{3}{|c|}{ Laboratory data set "b" } \\
\hline & \multicolumn{3}{|l|}{$H_{0}(\mathrm{~m})$} & \multicolumn{3}{|l|}{$H_{0}(\mathrm{~m})$} \\
\hline & 0.01 & 0.02 & 0.028 & 0.01 & 0.02 & 0.032 \\
\hline Measured & 0.047 & 0.043 & 0.039 & 0.115 & 0.095 & 0.084 \\
\hline Predicted & 0.0407 & 0.0399 & 0.039 & 0.0852 & 0.0842 & 0.0829 \\
\hline Error $(\%)$ & -13.40 & -7.21 & 0 & -25.91 & -11.37 & -1.31 \\
\hline
\end{tabular}

\section{Conclusions}

A 2DV numerical model based on an ALE description has been developed to simulate wave propagation over a fixed bed of cohesive mud layer by finite volume method. Nonlinear Navier-Stokes equations are solved by the use of projection method. The two-equation $k-\varepsilon$ turbulence model with buoyancy terms has been included in the numerical model. Bed fluidization and surface erosion are not considered, and mud bed is treated as a Newtonian fluid:

1. The application of small-amplitude Stokes wave train in deep water showed good agreements for water elevation, pressure field, and velocity distribution compared with the analytical solution.

2. The simulation of solitary wave propagation in constant water depth and comparing the predictions with analytical solutions of water elevation, pressure field, and velocity distribution showed the capability of the model in simulating non-linear terms, i.e., advection, in Navier-Stokes equations.

3. Simulation of non-linear short wave propagation in constant water depth and comparison of the predictions against measured values of free surface elevation confirmed the ability of the model in prediction of nonlinear short waves.

4. The model was applied to a two-layer system of water and fluid mud, and satisfactory results were obtained for attenuation coefficient of wave and its relationship with wave height and frequency in comparison with experimental data.

5. The model predictions show a milder trend for $k_{i}$ for smaller wave heights/larger viscosities compared with measured-based values.

6. Both laboratory data and numerical simulations reveal a decrease of wave attenuation rate with the increase of wave height.

7. Closer agreements for predicted-based wave damping coefficients with theoretical results compared with measured-based values, for higher frequencies, and the larger discrepancies for lower frequencies may be due to the non-Newtonian behavior of the mud layer for large frequencies.

8. The simulated values of the ratio of the interfacial to surface wave amplitude lie between the experimentalbased values and the theoretical solution.

9. The ratios of the interfacial to surface wave amplitudes are larger for mud of lower densities according to the theoretical-, numerical-, and experimental-based values, with the exception of experimental values for mud with a density of $1,300 \mathrm{~kg} / \mathrm{m}^{3}$.

10. The increase of mud layer thickness results in the decrease of surface wave number, which is in agreement with the theoretical solution.

In summary, the capability of the numerical model in simulation of non-linear short waves and a system of fluid mud were confirmed. Wave height, wave damping coefficient, and water-mud interface elevation were simulated and compared with the experimental data and theoretical solutions for various wave heights and frequencies. The results revealed a decrease of wave attenuation rate with an increase of wave height and in accordance with the measured values. The non-Newtonian behavior of mud layer, not being considered in the present formulation, may have affected the simulated values for large frequencies. In general, the ratios of the interfacial to surface wave amplitudes are larger for mud with a lower density, and the increase of mud layer thickness results in the decrease of surface wave number.

Open Access This article is distributed under the terms of the Creative Commons Attribution License which permits any use, distribution, and reproduction in any medium, provided the original author(s) and the source are credited.

\section{References}

Ahmadi A, Badiei P, Namin M (2007) An implicit two-dimensional non-hydrostatic model for free-surface flows. Int J Numer Methods Fluids 54(9):1055-1074

An NN (1993) Mud mass transport under wave and current. Ph.D. dissertation, Department of Civil Engineering, Yokohama National University, Yokohama

Brown DL (2001) Accuracy of projection methods for the incompressible Navier-Stokes equations. Workshop of numerical simulation of incompressible flows. US Department of Energy, California

Chapalain G, Cointe R, Temperville A (1992) Observed and modeled resonantly interacting progressive water-waves. J Coast Eng 16:267-300

Chorin AJ (1968) Numerical solution of the Navier-Stokes equations. Math Comput 22:745-762

Dalrymple RA, Liu PL-F (1978) Waves over soft muds, a two-layer fluid model. J Phys Oceanogr 8:1121-1131

De Wit PJ (1995) Liquifaction of cohesive sediment by waves. Ph.D. dissertation, Delft University of Technology, Delft

Dean RG, Dalrymple RA (1991) Water wave mechanics for engineers and scientists. Academic Series on Ocean Engineering, vol 2. World Scientific, Singapore 
Gade HG (1958) Effects of a non-rigid, impermeable bottom on plane surface waves in shallow water. J Mar Res 16:61-82

Golub GH, Van Loan CF (1989) Matrix computations, 2nd edn. The Johns Hopkins University Press, USA

Hejazi K (2005) 3D numerical modeling of flow and turbulence in oceanic water bodies using an ALE projection method. 1st Conference on Numerical Modeling in Civil Engineering, K.N. Toosi University of Tech

Hirt CW, Shannon JP (1968) Free-surface stress conditions for incompressible-flow calculations. J Comput Phys 2:403-411

Iwasaki T and Sato M (1972) Dissipation of wave energy due to opposing current. Proc. 13th Coastal Eng. Conf., ASCE, 1:605-622

Kaihatu JM, Sheremet A, Holland KT (2007) A model for the propagation of nonlinear surface waves over viscous muds. J Coast Eng $54: 752-764$

Kincaid D, Cheney W (1991) Numerical analysis. Brooks/Cole, USA

Li Y, Mehta A (1997). Mud fluidization by water waves. In: Burt N, Parker R, Watts J (eds) Cohesive sediments. Wiley, 341-363. Proc. 4th Nearshore and Estuarine Cohesive Sediment Transport Conference INTERCOH '94, Wallingford, England, UK.

Maa JP-Y, Mehta AJ (1990) Soft mud response to water waves. J Waterw Port Coast Ocean Eng ASCE 116(5):634-650

Mehta A, Williams D, Williams P, Feng J (1995) Tracking dynamical changes in mud bed due to waves. J Hydraul Eng 121(6):504-506

Mei CC (1983) The applied dynamics of ocean surface waves. Wiley Inter-science, New York

Ng C-O (2000) Water waves over a muddy bed: a two-layer Stokes' boundary layer model. J Coast Eng 40:221-242

$\mathrm{Ng} \mathrm{C-O} \mathrm{(2004)} \mathrm{Mass} \mathrm{transport} \mathrm{and} \mathrm{set-ups} \mathrm{due} \mathrm{to} \mathrm{partial} \mathrm{standing} \mathrm{surface}$ waves in a two-layer viscous system. J Fluid Mech 520:297-325
Niu X, Yu X (2010). A numerical model for wave propagation over muddy slope. ICCE, 32nd International Conference on Coastal Engineering, Shanghai, China.

Rodi W (1987) Examples of calculation models for flow and mixing in stratified fluids. J Geophysical Research 92(C5):5305-5328

Sakakiyama T, Bijker EW (1989) Mass transport velocity in mud layer due to progressive waves. J Waterw Port Coast Ocean Eng ASCE 115(5):614-633

Sorensen RM (1997) Basic coastal engineering. Kluwer Academic, New York

Tannehill JC, Anderson DA, Pletcher RH (1997) Computational fluid mechanics and heat transfer, 2nd edn. Taylor \& Francis, Washington

Temam R (1969) Sur l'Approximation de la Solution des Equations de Navier-Stokes par la Méthode des pas Fractionnaires (I): Archive for Rational Mechanics and Analysis, 32:135-153; (II): Archive for Rational Mechanics and Analysis, 33:377-385

Tsuruya H, Nakano S, Takahama J (1987) Interaction between surface waves and a multi-layered mud bed. Report of Port and Harbor Research Institute, Ministry of Transport, Japan, 26(5):138-173

Twizell EH (1984) Computational methods for partial differential equations. Horwood, Chichester

Weinan E, Jian-Guo L (1995) Projection method I: convergence and numerical boundary layers. SIAM J Numer Anal 32(4):1017-1057

Whitehouse R, Soulsby R, Roberts W and Mitchener H (2000) Dynamics of estuarine muds. Thomas Telford

Yuan HL, Wu CH (2004) A two-dimensional vertical non-hydrostatic $\sigma$ model with an implicit method for free-surface flows. Int J Numer Methods Fluids 44:811-835

Zhang D-H, Ng C-O (2006) A numerical study on wave-mud interaction. China Ocean Eng 20:383-394 